\title{
Stability of parabolic-hyperbolic traveling waves
}

\author{
Jens Rottmann-Matthes
}

Communicated by Carmen Chicone, received February 1, 2012.

\begin{abstract}
In this paper we investigate nonlinear stability of traveling waves in general parabolic-hyperbolic coupled systems where we allow for a nonstrictly hyperbolic part.

We show that the problem is locally well-posed in a neighborhood of the traveling wave and prove that nonlinear stability follows from stability of the point spectrum and a simple algebraic condition on the coefficients of the linearization. We also obtain rates of convergence that are directly related to the spectral gap. The proof is based on a trick to reformulate the PDE as a partial differential algebraic equation for which the zero eigenvalue is removed from the spectrum. Then the Laplace-technique becomes applicable and resolvent estimates are used to prove stability.

Our results apply to pulses as well as fronts and generalize earlier results of Bates and Jones [2] and of Kreiss, Kreiss, and Petersson [13]. As an example we present an application to the Hodgkin-Huxley model.
\end{abstract}

\section{Contents}

1. Introduction 30

2. Local Existence and Continuation 33

3. PDAE Reformulation 36

4. Spectral Properties of Linearizations 40

5. PDAE Stability 45

6. Nonlinear Stability of Traveling Waves 53

7. Application to the Hodgkin-Huxley Model 54

Appendix A. A Perturbation Result from Linear Algebra 57

$\begin{array}{lll}\text { Appendix B. Properties of Nonlinear Terms } & 59\end{array}$

Appendix C. Exponential Dichotomies 60

References $\quad 61$

1991 Mathematics Subject Classification. 35B40, 35M31, 35B35, 35P05.

Key words and phrases. Traveling waves, Parabolic-hyperbolic partial differential equations, Degenerate partial differential equations, Partial differential algebraic equations, Nonlinear stability, Asymptotic behavior, Resolvent estimates.

This research was supported by CRC 701 "Spectral Analysis and Topological Methods in Mathematics". 


\section{Introduction}

A prominent model from applications is the spatially extended Hodgkin-Huxley system [11], which models the signaling of electric pulses along nerve axons. The system is of reaction-diffusion type and has the feature that some of the components model ion channels which are spatially located and, therefore, do not diffuse. This leads to a parabolic equation coupled to a system of ordinary differential equations. The equations are of the form

$$
u_{t}=A u_{x x}+f_{1}(u, v), \quad v_{t}=f_{2}(u, v), \quad x \in \mathbb{R}, t \geq 0,
$$

where $u$ is a scalar function and $v$ is $\mathbb{R}^{3}$-valued. For the Hodgkin-Huxley equations the existence of traveling wave solutions is well-known. These are solutions of the form $u(x, t)=u^{o}\left(x-\lambda^{o} t\right), v(x, t)=v^{o}\left(x-\lambda^{o} t\right)$, where $\left(u^{o}, v^{o}\right)$ is the profile and $\lambda^{o}$ the speed of the wave. When the equation is considered in a co-moving frame with speed $\lambda^{o}$, that is, the new spatial variable $\xi=x-\lambda^{o} t$ is used, the equation becomes the coupled parabolic-hyperbolic equation

$$
u_{t}=A u_{\xi \xi}+\lambda^{o} u_{\xi}+f_{1}(u, v), \quad v_{t}=\lambda^{o} v_{\xi}+f_{2}(u, v),
$$

for which $\left(u^{o}, v^{o}\right)$ is a steady state.

In this paper we consider coupled parabolic-hyperbolic Cauchy problems of the following form that includes (1.2):

$$
\begin{gathered}
u_{t}=A_{11} u_{x x}+g(u, v)_{x}+f_{1}(u, v), \quad v_{t}=B_{22} v_{x}+f_{2}(u, v), \quad x \in \mathbb{R}, t \geq 0, \\
u(x, 0)=u_{0}(x) \in \mathbb{R}^{n}, \quad v(x, 0)=v_{0}(x) \in \mathbb{R}^{m} .
\end{gathered}
$$

By saying that (1.3) is parabolic-hyperbolic we understand that the following holds:

Assumption 1.1. (1) $A_{11} \in \mathbb{R}^{n, n}$ and $A_{11}+A_{11}^{T} \geq 2 \alpha I>0$ holds in the sense of Hermitian matrices,

(2) $B_{22} \in \mathbb{R}^{m, m}$ and $B_{22}=\operatorname{diag}\left(b_{1}, \ldots, b_{m}\right)$ is a diagonal matrix.

To include the Hodgkin-Huxley model, we allow $B_{22}$ to have multiple eigenvalues, i.e. the $v$-equation is assumed to be hyperbolic but not necessarily strictly hyperbolic. Compare this with $[\mathbf{1 3}]$, where strict hyperbolicity is assumed for the second equation.

We are interested in the asymptotic stability of traveling waves. Therefore, assume there exists a traveling solution of (1.3). We impose smoothness assumptions which are satisfied for many problems from applications, including the HodgkinHuxley model:

Assumption 1.2. The nonlinearities $f_{1}, f_{2}, g$ are of class $\mathcal{C}^{3}$, the profile $\left(u^{o}, v^{o}\right)$ of the traveling wave satisfies $u^{o} \in \mathcal{C}_{b}^{1}\left(\mathbb{R}, \mathbb{R}^{n}\right), u_{x}^{o} \in H^{2}\left(\mathbb{R}, \mathbb{R}^{n}\right)$, and $v^{o} \in \mathcal{C}_{b}^{1}\left(\mathbb{R}, \mathbb{R}^{m}\right)$, $v_{x}^{o} \in H^{2}\left(\mathbb{R}, \mathbb{R}^{m}\right)$, and, moreover, $f_{1}\left(u^{o}, v^{o}\right) \in L^{2}\left(\mathbb{R}, \mathbb{R}^{n}\right)$ and $f_{2}\left(u^{o}, v^{o}\right) \in L^{2}\left(\mathbb{R}, \mathbb{R}^{m}\right)$. $H^{1}$.

REMARK. The assumption implies $f_{i}\left(u^{o}, v^{o}\right) \in H^{2}$ for $i=1,2$ and $g\left(u^{o}, v^{o}\right)_{x} \in$

Throughout the paper we use the following notations: We write $\mathcal{C}_{b}^{k}$ for the space of $k$ times continuously differentiable and bounded functions, $L^{2}$ is the usual space of square integrable functions and for $k>0, H^{k}$ is the Sobolev space of $k$ times weakly differentiable functions whose distributional derivatives up to order $k$ can be represented by square integrable functions. We write $H^{-1}$ for the dual of $H^{1}$. To simplify notation we denote the evaluation of a function at $\left(u^{o}, v^{o}\right)$ by a 
superscript ${ }^{o}$, e.g. $g^{o}=g\left(u^{o}, v^{o}\right), g^{o}(x)=g\left(u^{o}(x), v^{o}(x)\right)$ etc. We also abbreviate $W(x, t)=\left(w_{1}(x, t), w_{2}(x, t)\right)^{T}=(u(x, t), v(x, t))^{T}$ and $W^{o}=\left(u^{o}, v^{o}\right)$.

Using these abbreviations, (1.3) reads

$$
W_{t}=A W_{x x}+G(W)_{x}+F(W), \quad W(x, 0)=W_{0}(x) \in \mathbb{R}^{n+m},
$$

where

$$
A=\left(\begin{array}{cc}
A_{11} & 0 \\
0 & 0
\end{array}\right), \quad G(W)=\left(\begin{array}{c}
g(W) \\
B_{22} w_{2}
\end{array}\right), \quad F(W)=\left(\begin{array}{c}
f_{1}(W) \\
f_{2}(W)
\end{array}\right), \text { and } W_{0}=\left(\begin{array}{l}
u_{0} \\
v_{0}
\end{array}\right) .
$$

A major difficulty in the proofs of wave stability is the shift equivariance of the equation. It is well-known that this leads to non-uniqueness because every solution gives rise to a whole one-parameter family of solutions, obtained by spatial shifts. Therefore, one cannot expect that a solution of the Cauchy problem converges to "the" traveling wave, but only to some shifted version of it, and one has to consider asymptotic stability with asymptotic phase. The equivariance also leads to a 0 eigenvalue of the linearized right hand side

$$
P W=A W_{x x}+\left(G_{W}^{o} W\right)_{x}+F_{W}^{o} W,
$$

which is a closed linear operator on $L^{2} \times L^{2}$ with domain $H^{2} \times H^{1}$.

Assumption 1.3. For the point spectrum $\sigma_{p t}(P)$ of the operator $P$ on $L^{2}$ holds $\sigma_{p t}(P) \cap\{\operatorname{Re} s>-\delta\}=\{0\}$ and 0 is a simple eigenvalue of $P$.

Define the matrices

$$
\begin{gathered}
A=\left(\begin{array}{cc}
A_{11} & 0 \\
0 & 0
\end{array}\right), \\
C(x)=\left(\begin{array}{cc}
C_{11}(x) & C_{12}(x) \\
C_{21}(x) & C_{22}(x)
\end{array}\right)=\left(\begin{array}{cc}
g_{u x}^{o}(x)+f_{1, u}^{o}(x) & g_{12}^{o}(x) \\
0 & B_{22}(x)+f_{1, v}^{o}(x) \\
f_{2, u}^{o}(x) & f_{2, v}^{o}(x)
\end{array}\right),
\end{gathered}
$$

where a subindex $u$ (or $v$ ) denotes the partial derivative with respect to the $u$ (or $v)$ variables. Then the operator $P$ can be written as

$$
P W=A W_{x x}+B W_{x}+C W .
$$

For the analysis of asymptotic stability of traveling waves we use the techniques developed in [19] for first-order hyperbolic systems: Write $W(x, t)$, the solution of (1.4), as

$$
W(x, t)=W^{o}(x-\widetilde{\varphi}(t))+\widetilde{W}(x, t) .
$$

This introduces new nonlinear coordinates $(\widetilde{W}, \widetilde{\varphi})$ and increases the degrees of freedom by one. To make this change of coordinates unambiguous, the function $\widetilde{W}$ is assumed to lie in the kernel of some suitable linear functional $\Psi$, i.e. $\Psi(\widetilde{W}(t))=0$ for all $t$. This leads to the partial differential algebraic equation (PDAE)

$$
\begin{aligned}
\widetilde{W}_{t} & =A\left(W^{0}(\cdot-\widetilde{\varphi})+\widetilde{W}\right)_{x x}+G\left(W^{0}(\cdot-\widetilde{\varphi})+\widetilde{W}\right)_{x} \\
& +F\left(W^{0}(\cdot-\widetilde{\varphi})+\widetilde{W}\right)+W_{x}^{0}(\cdot-\widetilde{\varphi}) \widetilde{\lambda} \\
\widetilde{\varphi}_{t} & =\widetilde{\lambda} \\
0 & =\Psi(W)
\end{aligned}
$$

for $\widetilde{W}, \widetilde{\varphi}$, and $\widetilde{\lambda}$. In Section 3 we show that the change of coordinates (1.7) is indeed well-defined under very general assumptions on $\Psi$. Note that this approach 
is closely related to the technique used by Henry in $[\mathbf{1 0}$, Ch. 5], but there the theory of analytic semigroups is heavily used.

Another commonly used approach is to use Riesz projectors to project the equation onto the subspace orthogonal to the eigenfunction, see for example $[\mathbf{9}$, 13]. The projection approach seems to be more difficult to generalize when the 0 -eigenvalue is part of the essential spectrum and it also seems to be less straight forward to prove well-posedness for the projected equation when one is not in the setting of analytic semigroups. Also note that the semigroup approach chosen in [9] does not apply in our situation because of the nonlinear advection term $g(u, v)_{x}$.

The plan for the rest of the paper is as follows. In Section 2 we show wellposedness of an abstract formulation of Cauchy problem (1.3). The abstract formulation allows to apply the result in Section 5 directly to a projected PDE. Of course, there is a large amount of literature concerned with the problem of existence and well-posedness for coupled parabolic-hyperbolic systems. In particular, we mention the results of Vol'pert and Hudjaev [23], the monograph [14] by Kreiss and Lorenz, and results by Zheng and co-authors, e.g. the monograph [27] and the references therein. But none of these results applies in our setting since they all are concerned with perturbations of rest states so that initial data close to a traveling front do not seem to be included.

Section 4 is concerned with the spectral properties of the linear operator $P$ and the linearization of the PDAE. We show uniform resolvent estimates for the PDAE in an open half plane that includes the imaginary axis. We give a simple spectral Assumption (see Assumption 4.1 3) which unifies the assumptions made on the asymptotic coefficients of $P$ as $|x| \rightarrow \infty$. See also [13], Assumption 1 (parabolic part) and Assumption 2 (hyperbolic part), which both have to be used in the proof of Theorem 4.1 from that paper. An important tool for the derivation of estimates from the spectral assumption is a perturbation result for invariant subspaces, which is recalled in Appendix A for convenience.

In Section 5 we show exponential well-posedness of the PDAE problem which justifies application of the Laplace transform. Then Plancherels Theorem is used to translate resolvent estimates for the PDAE-problem to linear stability properties of the PDAE. A bootstrapping argument then shows also nonlinear stability of the PDAE.

In Section 6 we use the relation of the PDE to the PDAE problem, obtained in Section 3, to prove our main stability result for traveling waves:

THEOREM 1.4 (Asymptotic stability of traveling waves). Let $\left(u^{o}, v^{o}\right)$ be a traveling wave solution of (1.3) with speed $\lambda^{\circ}$. Assume that Assumptions 1.1, 1.2, 1.3, 4.1 hold for the co-moving equation

$$
\begin{array}{ll}
u_{t}=A_{11} u_{x x}+g(u, v)_{x}+f_{1}(u, v)+\lambda^{o} u_{x}, & u(0)=u_{0}, \\
v_{t}=B_{22} v_{x}+f_{2}(u, v)+\lambda^{o} v_{x}, & v(0)=v_{0} .
\end{array}
$$

Then for every $0<\eta<\delta$ there is $\rho>0$ so that for all initial data $u_{0} \in u^{o}+H^{2}$ and $v_{0} \in v^{o}+H^{2}$, with $\left(\left\|u_{0}-u^{o}\right\|_{H^{2}}^{2}+\left\|v_{0}-v^{o}\right\|_{H^{2}}^{2}\right)^{1 / 2}<\rho$, exists a unique solution $(u, v)$ of $(1.3)$ on $[0, \infty)$. The solution satisfies smoothness properties

$$
\begin{aligned}
& u \in \mathcal{C}\left([0, T] ; u^{o}+H^{1}\right) \cap H^{1}\left(0, T ; u^{o}+L^{2}\right) \cap L^{2}\left(0, T ; u^{o}+H^{2}\right), \\
& v \in \mathcal{C}\left([0, T] ; v^{o}+H^{1}\right) \cap H^{1}\left(0, T ; v^{o}+L^{2}\right) .
\end{aligned}
$$


Moreover, there is $\varphi_{\infty}=\varphi_{\infty}\left(u_{0}, v_{0}\right) \in \mathbb{R}$ and a constant $C=C(\eta)>0$, independent of $\left(u_{0}, v_{0}\right)$, so that

$$
\begin{gathered}
\left|\varphi_{\infty}\right| \leq C\left(\left\|u_{0}-u^{o}\right\|_{H^{2}}+\left\|v_{0}-v^{o}\right\|_{H^{2}}\right), \quad \text { and } \\
\left\|u(t)-u^{o}\left(\cdot-\lambda^{o} t-\varphi_{\infty}\right)\right\|_{H^{1}}+\left\|v(t)-v^{o}\left(\cdot-\lambda^{o} t-\varphi_{\infty}\right)\right\|_{H^{1}} \\
\leq C\left(\left\|u_{0}-u^{o}\right\|_{H^{2}}+\left\|v_{0}-v^{o}\right\|_{H^{2}}\right) e^{-\eta t} \quad \forall t \geq 0 .
\end{gathered}
$$

We finish this paper with an application of our results to the important HodgkinHuxley model from biology in Section 7. For the application to a traveling front in the FitzHugh-Nagumo equation and numerical experiments, where the predicted rates can be observed numerically, we refer to $[\mathbf{2 1}]$.

Acknowledgment: The author would like to thank Prof. Wolf-Jürgen Beyn for many helpful discussions and the supervision of the $\mathrm{PhD}$ thesis $[\mathbf{1 8}]$ in which many of the results from this paper were obtained.

\section{Local Existence and Continuation}

In this section we consider (global) existence and uniqueness of solutions to the nonlinear problem (1.3) with the general result given in the Theorem 2.5 and its application to traveling waves in Theorem 2.8. As mentioned above, various parabolic-hyperbolic problems have been analyzed in the literature. But because none of the results applies to our situation, we present some results obtained in the author's PhD thesis [18]. Since the techniques used are quite standard, we concentrate on the ideas and refer to [18] for the details.

By considering the equation in a co-moving frame, we may assume $\lambda^{o}=0$ without loss of generality. Moreover, instead of (1.3) we consider the following generalized problem which makes the results easily applicable also to a projected problem in Section 5:

$$
\begin{aligned}
u_{t} & =A_{11} u_{x x}+\widetilde{g}(u, v)_{x}+\widetilde{f}_{1}(u, v), & & u(0)=u_{0} \in H^{1}, \\
v_{t} & =B_{22} v_{x}+\widetilde{f}_{2}(u, v), & v(0) & =v_{0} \in H^{1} .
\end{aligned}
$$

Here $\widetilde{g}, \widetilde{f}_{1}, \widetilde{f}_{2}$ are (abstract) nonlinear operators on which we impose

Assumption 2.1. For all $0 \leq t_{0}<T, u, u^{\prime} \in L^{2}\left(t_{0}, T ; H^{1}\left(\mathbb{R}, \mathbb{R}^{n}\right)\right) \cap L^{\infty}\left(t_{0}, T ; H^{1}\right)$ and $v, v^{\prime} \in L^{2}\left(t_{0}, T ; H^{1}\left(\mathbb{R}, \mathbb{R}^{m}\right)\right) \cap L^{\infty}\left(t_{0}, T ; H^{1}\right)$ the operators $\widetilde{g}, \widetilde{f}_{1}$, and $\widetilde{f}_{2}$ satisfy:

(1) $\widetilde{g}(u, v), \widetilde{f}_{1}(u, v) \in L^{2}\left(t_{0}, T ; H^{1}\left(\mathbb{R}, \mathbb{R}^{n}\right)\right)$ and $\widetilde{f}_{2}(u, v) \in L^{2}\left(t_{0}, T ; H^{1}\left(\mathbb{R}, \mathbb{R}^{m}\right)\right)$,

(2) there is $C=C(T)>0$, independent of $t_{0}$, so that $\left(0 \in L^{2}\left(t_{0}, T ; H^{1}\right) \cap\right.$ $\left.L^{\infty}\left(t_{0}, T ; H^{1}\right)\right)$

$$
\left\|\widetilde{f}_{1}(0,0)\right\|_{L^{2}\left(t_{0}, T ; H^{1}\right)}+\|\widetilde{g}(0,0)\|_{L^{2}\left(t_{0}, T ; H^{1}\right)}+\left\|\widetilde{f}_{2}(0,0)\right\|_{L^{2}\left(t_{0}, T ; H^{1}\right)} \leq C
$$

(3) for all $T, K>0$ exists $C>0$, independent of $t_{0}$, so that if $\|*\|_{L^{\infty}\left(t_{0}, T ; H^{1}\right)} \leq$ $K$ for all $* \in\left\{u, u^{\prime}, v, v^{\prime}\right\}$ holds for $\widetilde{h} \in\left\{\widetilde{g}, \widetilde{f}_{1}, \widetilde{f}_{2}\right\}$

(2.2) $\left\|\widetilde{h}(u, v)-\widetilde{h}\left(u^{\prime}, v^{\prime}\right)\right\|_{L^{2}\left(t_{0}, T ; H^{1}\right)}^{2} \leq C\left(\left\|u-u^{\prime}\right\|_{L^{2}\left(t_{0}, T ; H^{1}\right)}^{2}+\left\|v-v^{\prime}\right\|_{L^{2}\left(t_{0}, T ; H^{1}\right)}^{2}\right)$.

LEMma 2.2. (1) Let Assumption 1.2 hold and $g_{l}, f_{1, l}, f_{2, l} \in L_{l o c}^{2}\left([0, \infty) ; H^{1}\right)$.

Then the following Nemytskii operators satisfy Assumption 2.1:

$$
\begin{aligned}
\widetilde{g}(u, v) & =g\left(u^{o}+u, v^{o}+v\right)-g\left(u^{o}, v^{o}\right)+g_{l}(x, t), \\
\widetilde{f}_{i}(u, v) & =f_{i}\left(u^{o}+u, v^{o}+v\right)-f_{i}\left(u^{o}, v^{o}\right)+f_{i, l}(x, t), \quad i=1,2 .
\end{aligned}
$$


(2) Let $B_{11}, C_{11} \in \mathcal{C}_{b}^{1}\left(\mathbb{R}, \mathbb{R}^{n, n}\right), B_{12}, C_{12} \in \mathcal{C}_{b}^{1}\left(\mathbb{R}, \mathbb{R}^{n, m}\right), B_{1 j, x} \in H^{1}, C_{21} \in$ $\mathcal{C}_{b}^{1}\left(\mathbb{R}, \mathbb{R}^{m, n}\right), C_{22} \in \mathcal{C}_{b}^{1}\left(\mathbb{R}, \mathbb{R}^{m, m}\right), g_{l}, f_{1, l}, f_{2, l} \in L_{l o c}^{2}\left([0, \infty) ; H^{1}\right)$. Then Assumption 2.1 holds for

$$
\begin{aligned}
\widetilde{g}(u, v) & =B_{11}(x) u+B_{12}(x) v+g_{l}(x, t), \\
\tilde{f}_{i}(u, v) & =C_{i 1}(x) u+C_{i 2}(x) v+f_{i, l}(x, t), \quad i=1,2 .
\end{aligned}
$$

Because we are interested in perturbations of steady states, we use the new dependent variables $\widetilde{u}=u-u^{o}$ and $\widetilde{v}=v-v^{o}$ to rewrite equation (1.3) in the form $(2.1)$

$$
\begin{array}{ll}
\widetilde{u}_{t}=A_{11} \widetilde{u}_{x x}+\widetilde{g}(\widetilde{u}, \widetilde{v})_{x}+\widetilde{f}_{1}(\widetilde{u}, \widetilde{v}), & \widetilde{u}(0)=u_{0}-u^{o} \in H^{1}, \\
\widetilde{v}_{t}=B_{22} \widetilde{v}_{x}+\widetilde{f}_{2}(\widetilde{u}, \widetilde{v}), & \widetilde{v}(0)=v_{0}-v^{o} \in H^{1} .
\end{array}
$$

Here $\widetilde{g}(\widetilde{u}, \widetilde{v})=g\left(u^{o}+\widetilde{u}, v^{o}+\widetilde{v}\right)-g\left(u^{o}, v^{o}\right)$ and $\widetilde{f}_{i}(\widetilde{u}, \widetilde{v})=f_{i}\left(u^{o}+\widetilde{u}, v^{o}+\widetilde{v}\right)-f_{i}\left(u^{o}, v^{o}\right)$ for $i=1,2$. By Lemma 2.2 equation (2.3) satisfies Assumption 2.1.

We use the following solution concept, adapted from $[8, \S 7.1,7.3$ and 9.2] to our situation.

DEFinition 2.3 (Weak solution). We call $(u, v)$ a weak solution of (2.1) on $[0, T]$ if

(0) $u \in L^{2}\left(0, T ; H^{1}\left(\mathbb{R}, \mathbb{R}^{n}\right)\right) \cap L^{\infty}\left(0, T ; H^{1}\right)$ with $u_{t} \in L^{2}\left(0, T ;\left(H^{1}\right)^{\prime}\right)$, $v \in L^{2}\left(0, T ; H^{1}\left(\mathbb{R}, \mathbb{R}^{m}\right)\right) \cap L^{\infty}\left(0, T ; H^{1}\right)$ with $v_{t} \in L^{2}\left(0, T ; L^{2}\right)$,

(1) $u(0)=u_{0}$ and for all $\phi \in H^{1}\left(\mathbb{R}, \mathbb{R}^{n}\right)$ and a.e. $t \in[0, T]$ holds

$$
\left\langle u_{t}, \phi\right\rangle+\int_{\mathbb{R}}\left(A_{11} u_{x}\right)^{T} \phi_{x}-\left(\widetilde{g}(u, v)_{x}\right)^{T} \phi-\left(\tilde{f}_{1}(u, v)\right)^{T} \phi d x=0,
$$

here $\langle\cdot, \cdot\rangle$ denotes the duality pairing of $H^{1}$ and $H^{-1}=\left(H^{1}\right)^{\prime}$,

(2) $v(0)=v_{0}$ and for all $\phi \in H^{1}\left(\mathbb{R}, \mathbb{R}^{m}\right)$ and a.e. $t \in[0, T]$ holds

$$
\left\langle v_{t}, \phi\right\rangle_{L^{2}}-\int_{\mathbb{R}}\left(B_{22} v_{x}\right)^{T} \phi+\left(\widetilde{f}_{2}(u, v)\right)^{T} \phi d x=0 .
$$

It is called a weak solution on $\left[0, T^{*}\right), T^{*} \in(0, \infty]$, if it is a weak solution on $[0, T]$ for all $0<T<T^{*}$.

The assumption $u, v \in L^{\infty}\left(0, T ; H^{1}\right)$ is needed for the Lipschitz bound (2.2) of the nonlinear operators in (2.3), which turns out to be to be important for uniqueness of weak solutions, see $[\mathbf{1 8}, \S 4.1]$.

Note that the initial conditions are reasonable because of Sobolev embedding. In fact, one can prove that the weak solution even is a strong solution in the following sense:

Definition 2.4 (Strong solution). A strong solution of $(2.1)$ on $[0, T]$ is a weak solution satisfying $u \in L^{2}\left(0, T ; H^{2}\right) \cap H^{1}\left(0, T ; L^{2}\right)$ and $v \in L^{2}\left(0, T ; H^{1}\right) \cap$ $H^{1}\left(0, T ; L^{2}\right)$ so that $(2.1 \mathrm{a})$ and $(2.1 \mathrm{~b})$ hold as equalities in $L^{2}\left(0, T ; L^{2}\right)$. It is called a strong solution on $\left[0, T^{*}\right), T^{*} \in(0, \infty]$, if it is a strong solution on $[0, T]$ for all $0<T<T^{*}$.

Now we can state the local existence and uniqueness result.

ThEOREM 2.5 (Existence and uniqueness). Impose Assumptions 1.1 and 2.1. Then there is $T^{*} \in(0, \infty]$ and a strong solution $\left(u^{*}, v^{*}\right)$ of the Cauchy problem (2.1) on $\left[0, T^{*}\right)$. If $(u, v)$ is a weak solution of $(2.3)$ on $[0, T]$ for some $T>0$, then 
$T<T^{*}$ and $u=\left.u^{*}\right|_{[0, T]}, v=\left.v^{*}\right|_{[0, T]}$, where the equalities hold in $L^{\infty}\left(0, T ; H^{1}\right)$. Moreover,

$$
\begin{aligned}
& u^{*} \in \mathcal{C}\left([0, T] ; H^{1}\right) \cap H^{1}\left(0, T ; L^{2}\right) \cap L^{2}\left(0, T ; H^{2}\right), \\
& v^{*} \in \mathcal{C}\left([0, T] ; H^{1}\right) \cap H^{1}\left(0, T ; L^{2}\right)
\end{aligned}
$$

for all $0<T<T^{*}$ and it holds the dichotomy

$$
\text { either } \quad T^{*}=+\infty \quad \text { or } \quad T^{*}<\infty \text { and } \lim _{t \nearrow T^{*}}\|u(t)\|_{H^{1}}+\|v(t)\|_{H^{1}}=+\infty .
$$

OutLINE OF THE PROOF. We do not give a full proof of the theorem, which can be found in $[\mathbf{1 8}, \S 4.1]$. Instead we only outline the basic steps.

The principal idea is to treat the parabolic and hyperbolic parts separately and to couple them by an iteration process. This idea also appears in [25], where it is used to show existence for a biologically motivated system, with a wave type hyperbolic part.

To formalize the idea, let $U_{T}=\mathcal{C}\left([0, T] ; H^{1}\left(\mathbb{R}, \mathbb{R}^{n}\right)\right),\|u\|_{U_{T}}:=\|u\|_{L^{\infty}\left(0, T ; H^{1}\right)}$, and $V_{T}=\mathcal{C}\left([0, T] ; H^{1}\left(\mathbb{R}, \mathbb{R}^{m}\right)\right),\|v\|_{V_{T}}:=\|v\|_{L^{\infty}\left(0, T ; H^{1}\right)}$, and define the operator (which will be iterated)

$$
\mathcal{S}: U_{T} \times V_{T} \rightarrow U_{T} \times V_{T}, \quad\left(u^{k}, v^{k}\right) \mapsto \mathcal{S}\left(u^{k}, v^{k}\right):=\left(u^{k+1}, v^{k+1}\right) .
$$

Here $u^{k+1}$ is the unique weak solution of the linear parabolic Cauchy problem

$$
u_{t}=A_{11} u_{x x}+\widetilde{g}\left(u^{k}, v^{k}\right)_{x}+\widetilde{f}_{1}\left(u^{k}, v^{k}\right), \quad u(0)=u_{0},
$$

and $v^{k+1}$ is given as the unique weak solution of the linear hyperbolic Cauchy problem

$$
v_{t}=B_{22} v_{x}+\widetilde{f}_{2}\left(u^{k+1}, v^{k}\right), \quad v(0)=v_{0} .
$$

The proof of Theorem 2.5 then proceeds in the following steps

(1) Show well-posedness and derive solution estimates for (2.7) in terms of $u^{k}, v^{k}$.

(2) Show well-posedness and derive solution estimates for (2.8) in terms of $u^{k+1}, v^{k}$.

(3) Couple these estimates to show that the solution operator $\mathcal{S}$ is well-defined and a contraction for sufficiently small $T>0$. Because a fixed point of $\mathcal{S}$ is a solution of the coupled parabolic-hyperbolic PDE (2.1), solvability follows.

(4) Show uniqueness of the weak solution.

(5) Extend the solution as long as it stays bounded to obtain (2.5).

The following energy estimate can be used to prove global well-posedness for linear equations and will also be applied in the stability estimates. Note that the estimate is in integral form. This is important because we will be able to bound time integrals of the solution by time integrals of the data, using the Laplace technique (see 5.1.2).

Lemma 2.6 ([18, Lemma E.2]). Let Assumption 1.1 hold and impose the assumptions from Lemma 2.2 2. Then for every $\eta_{0} \geq 0$ there exists $C>0$ so that 
for all $\eta \leq \eta_{0}$ and all $u_{0} \in H^{1}, v_{0} \in H^{1}$ the strong solution of (2.1) with $\widetilde{g}$ and $\widetilde{f}_{i}$ from Lemma 2.2 2., given by Theorem 2.5, satisfies for all $t \geq 0$

$$
\begin{aligned}
& e^{2 \eta t}\left(\|u(t)\|_{H^{1}}^{2}+\|v(t)\|_{H^{1}}^{2}\right) \\
& \leq\left\|u_{0}\right\|_{H^{1}}^{2}+\left\|v_{0}\right\|_{H^{1}}^{2}+C \int_{0}^{t} e^{2 \eta \tau}\left(\|u(\tau)\|_{H^{1}}^{2}+\|v(\tau)\|_{H^{1}}^{2}\right) d \tau \\
& \quad+C \int_{0}^{t} e^{2 \eta \tau}\left(\left\|g_{l}(\tau)\right\|_{H^{1}}^{2}+\left\|f_{1, l}(\tau)\right\|_{L^{2}}^{2}+\left\|f_{2, l}(\tau)\right\|_{H^{1}}^{2}\right) d \tau .
\end{aligned}
$$

The assertion of the lemma easily follows for smooth functions and inhomogeneities. The general case is obtained by approximation. A proof, using a mollification trick, is given in [18, Appendix E].

Theorem 2.5 combined with the energy estimate from Lemma 2.6 shows global existence and exponential well-posedness for the inhomogeneous linear case:

Corollary 2.7 (Linear well-posedness of parabolic-hyperbolic coupled PDEs). Let the assumptions of Lemma 2.6 hold. Then for every $u_{0}, v_{0} \in H^{1}$ there is a unique strong solution $(u, v)$ on $[0, \infty)$ of $(2.1)$, which is unique also in the class of weak solutions. Furthermore, for every $\eta_{0} \geq 0$ there are constants $k, c \in \mathbb{R}$ so that for every $\eta \in \mathbb{R}$ with $\eta \leq \eta_{0}$ and all $0 \leq t<\infty$ the solution is bounded by

$\left\|\left(\begin{array}{c}u(t) \\ v(t)\end{array}\right)\right\|_{H^{1}}^{2} \leq k e^{c t}\left[\left\|\left(\begin{array}{c}u_{0} \\ v_{0}\end{array}\right)\right\|_{H^{1}}^{2}+\int_{0}^{t} e^{2 \eta \tau}\left(\left\|g_{l}(\tau)\right\|_{H^{1}}^{2}+\left\|f_{1, l}(\tau)\right\|_{L^{2}}^{2}+\left\|f_{2, l}(\tau)\right\|_{H^{1}}^{2}\right) d \tau\right]$.

Another immediate consequence of Theorem 2.5 is unique solvability of (1.3) in a neighborhood of the traveling wave $\left(u^{o}, v^{o}\right)$, note that we still require $\lambda^{o}=0$ without loss of generality.

ThEOREM 2.8 (Existence and uniqueness). Impose Assumptions 1.1 and 1.2 with $\lambda^{o}=0$.

Then for every initial data $u_{0} \in u^{o}+H^{1}\left(\mathbb{R}, \mathbb{R}^{n}\right), v_{0} \in v^{o}+H^{1}\left(\mathbb{R}, \mathbb{R}^{m}\right)$, there is a unique global solution of $(1.3)$, i.e. there is $T^{*} \in(0, \infty]$ and $\left(u^{*}, v^{*}\right)$ so that for all $0<T<T^{*}$ holds

$$
\begin{aligned}
& u^{*} \in \mathcal{C}\left([0, T] ; u^{o}+H^{1}\right) \cap H^{1}\left(0, T ; u^{o}+L^{2}\right) \cap L^{2}\left(0, T ; u^{o}+H^{2}\right), \\
& v^{*} \in \mathcal{C}\left([0, T] ; v^{o}+H^{1}\right) \cap H^{1}\left(0, T ; v^{o}+L^{2}\right),
\end{aligned}
$$

and $\left(u^{*}, v^{*}\right)$ is the unique solution of $(1.3)$ on $[0, T]$, where (1.3) holds as an equality in $L^{2}\left(\mathbb{R}, \mathbb{R}^{n}\right) \times L^{2}\left(\mathbb{R}, \mathbb{R}^{m}\right)$ for almost every $t \in[0, T]$. Moreover,

$$
\begin{aligned}
& \text { either } T^{*}=+\infty \text { or } \\
& 0<T^{*}<+\infty \text { and } \lim _{t \nearrow T^{*}}\left\|u^{*}(t)-u^{o}\right\|_{H^{1}}+\left\|v^{*}(t)-v^{o}\right\|_{H^{1}}=+\infty
\end{aligned}
$$

\section{PDAE Reformulation}

Let the setting be as in the introduction. By considering (1.3) in a co-moving frame we may assume $\lambda^{o}=0$ throughout this section. In this section we rigorously justify the change of coordinates (1.7) and give a precise meaning to the nonlinear PDAE (1.8). In particular, we show how solutions of the original problem (1.4) are related to solutions of the PDAE (1.8) and vice versa. To make the change of coordinates (1.7) unambiguous we impose on $\Psi$ : 
Assumption 3.1. The functional $\Psi$ acts on $H^{-1}\left(\mathbb{R}, \mathbb{R}^{n}\right) \times H^{-1}\left(\mathbb{R}, \mathbb{R}^{m}\right)$, is of the form $\Psi(u, v)=\psi_{1}(u)+\psi_{2}(v)$, and there is $C_{\Psi}>0$ so that

$$
|\Psi(u, v)| \leq C_{\Psi}\left(\|u\|_{H^{-1}}+\|v\|_{H^{-1}}\right), \quad \forall u \in H^{-1}\left(\mathbb{R}, \mathbb{R}^{n}\right), v \in H^{-1}\left(\mathbb{R}, \mathbb{R}^{m}\right) .
$$

Furthermore, $\Psi$ satisfies the non-degeneracy condition

$$
\Psi\left(u_{x}^{o}, v_{x}^{o}\right)=\psi_{1}\left(u_{x}^{o}\right)+\psi_{2}\left(v_{x}^{o}\right) \neq 0 .
$$

Of course, by linear continuation, $\Psi$ can also be considered as a linear functional on the respective complex-valued Sobolev-spaces. Note that two functions $\eta_{1} \in$ $H^{1}\left(\mathbb{R}, \mathbb{R}^{n}\right)$ and $\eta_{2} \in H^{1}\left(\mathbb{R}, \mathbb{R}^{m}\right)$ define a linear functional on $H^{-1} \times H^{-1}$ with (3.1) via $\Psi(u, v):=\left(u, \eta_{1}\right)_{L^{2}}+\left(v, \eta_{2}\right)_{L^{2}}$. Here $(-,-)_{L^{2}}$ denotes the $L^{2}$-inner product.

The following lemma, which easily follows from (3.2) with the inverse function theorem, is the key to local well-definedness of (1.7).

LEMMA 3.2. Let $\Psi$ be given as above. Then there are open neighborhoods $U, V$ of 0 in $\mathbb{R}$ so that the mapping $E: U \rightarrow V$ defined by $E(\widetilde{\varphi})=\Psi\left(W^{o}(\cdot-\widetilde{\varphi})-W^{o}\right)$ is a $\mathcal{C}^{2}$-diffeomorphism.

For $E: U \rightarrow V$ as in Lemma 3.2 define $\Phi$ by $\Phi:=E^{-1}: V \rightarrow U$, and let $\Xi$ and $\Upsilon$ denote the following nonlinear changes of coordinates:

$$
\Xi\left(\begin{array}{c}
\widetilde{W} \\
\widetilde{\varphi}
\end{array}\right):=\widetilde{W}+W^{o}(\cdot-\widetilde{\varphi}), \quad \Upsilon(W):=\left(\begin{array}{c}
W-W^{o}\left(\cdot-\Phi\left(\Psi\left(W-W^{o}\right)\right)\right) \\
\Phi\left(\Psi\left(W-W^{o}\right)\right)
\end{array}\right) .
$$

A simple computation shows that $\Xi$ with domain $\mathcal{D}_{\Xi}$ and $\Upsilon$ with domain $\mathcal{D}_{\Upsilon}$ are inverse to each other, where

$$
\begin{aligned}
& \mathcal{D}_{\Xi}:=\left\{(\widetilde{W}, \widetilde{\varphi}) \in L^{2}\left(\mathbb{R}, \mathbb{R}^{n+m}\right) \times \mathbb{R}: \widetilde{\varphi} \in U, \Psi(\widetilde{W})=0\right\}, \text { and } \\
& \mathcal{D}_{\Upsilon}:=\left\{W \in W^{o}+L^{2}\left(\mathbb{R}, \mathbb{R}^{n+m}\right): \Psi\left(W-W^{o}\right) \in V\right\} .
\end{aligned}
$$

Now we apply the change of coordinates $\Upsilon$ to solutions of (1.3).

LEMMA 3.3. Let the setting be as above and let $W=(u, v)$ be the unique global solution of (1.3) on $\left[0, T^{*}\right)$, given by Theorem 2.8. Assume there is $0<T<T^{*}$ so that $\mu(t):=\Psi\left(W(t)-W^{o}\right) \in V$ for all $t \in[0, T]$. Then $(\widetilde{u}, \widetilde{v}, \widetilde{\varphi})=\Upsilon(W)$ satisfies

$$
\begin{aligned}
\widetilde{u} & \in \mathcal{C}\left([0, T] ; H^{1}\right) \cap H^{1}\left(0, T ; L^{2}\right) \cap L^{2}\left(0, T ; H^{2}\right), \\
\widetilde{v} & \in \mathcal{C}\left([0, T] ; H^{1}\right) \cap H^{1}\left(0, T ; L^{2}\right), \\
\widetilde{\varphi} & \in \mathcal{C}^{1}([0, T] ; \mathbb{R}),
\end{aligned}
$$

and $\Psi(\widetilde{u}(t), \widetilde{v}(t))=0$ for all $t \in[0, T]$.

Proof. By Theorem 2.8, $t \mapsto W(t)-W^{o}$ is an element of $\mathcal{C}\left([0, T] ; H^{1}\right)$, so that $\mu \in \mathcal{C}([0, T] ; \mathbb{R})$ and the assumption $\mu(t) \in V$ is reasonable for $W(0)$ close to $W^{o}$. Moreover, $t \mapsto W(t)-W^{o} \in H^{1}\left(0, T ; L^{2}\right)$, so that $\mu \in L^{2}(0, T ; \mathbb{R})$ and $\Psi\left(W_{t}\right) \in L^{2}(0, T ; \mathbb{R})$ by properties of the Bochner integral (e.g. [26, Ch. 5]) since $\Psi$ is also a continuous linear functional on $L^{2}$. It easily follows $\mu_{t}=\Psi\left(W_{t}\right)$ in the sense of Distributions, so that $\mu \in H^{1}(0, T ; \mathbb{R})$.

Because $(u, v)$ solves (1.3),

$$
\mu_{t}=\psi_{1}\left(A_{11} u_{x x}+g(u, v)_{x}+f_{1}(u, v)\right)+\psi_{2}\left(B_{22} v_{x}+f_{2}(u, v)\right)
$$


holds as an equality in $L^{2}(0, T ; \mathbb{R})$. In fact, it follows that there is a continuous representative of $\mu_{t}$, i.e. $\mu \in \mathcal{C}^{1}$, since Theorem 2.8 implies (see also Corollary B.3)

$$
\begin{gathered}
\left(u-u^{o}\right)_{x x} \in \mathcal{C}\left([0, T] ; H^{-1}\right), \quad\left(v-v^{o}\right)_{x} \in \mathcal{C}\left([0, T] ; H^{-1}\right), \\
\left(g(u, v)-g^{o}\right)_{x} \in \mathcal{C}\left([0, T] ; L^{2}\right) \\
f_{1}(u, v)-f_{1}^{o}, f_{2}(u, v)-f_{2}^{o} \in \mathcal{C}\left([0, T] ; L^{2}\right)
\end{gathered}
$$

This proves $\widetilde{\varphi}=\Phi \circ \mu \in \mathcal{C}^{1}([0, T] ; \mathbb{R})$. To see that $\widetilde{u}$ is of the asserted class, write it as $\widetilde{u}=\left(u-u^{o}\right)+\left(u^{o}-u^{o}(\cdot-\widetilde{\varphi})\right)$. By Theorem 2.8 the first summand is of the correct class. The second summand belongs to $\mathcal{C}^{1}\left([0, T] ; H^{1}\right) \cap \mathcal{C}^{0}\left([0, T] ; H^{2}\right)$ by Assumption 1.2. The same arguments work for $\widetilde{v}$.

Finally, $\widetilde{W}=(\widetilde{u}, \widetilde{v}) \in \mathcal{N}(\Psi)$ follows from the definintion of $\widetilde{\varphi}$ :

$\Psi(\widetilde{W})=\Psi\left(W-W^{o}(\cdot-\widetilde{\varphi})\right)=\Psi\left(W-W^{o}\right)+\Psi\left(W^{o}-W^{o}(\cdot-\widetilde{\varphi})\right)=\mu-G(\Phi(\mu))=0$

Now assume that $W=(u, v)$ solves (1.3) and satisfies $\Psi\left(W(t)-W^{o}\right) \in V$ for all $t \in[0, T]$. Let $(\widetilde{W}, \widetilde{\varphi})=\Upsilon(W)$, i.e. $W=\widetilde{W}+W^{o}(\cdot-\widetilde{\varphi})$, and define $\widetilde{\lambda}=\widetilde{\varphi}_{t}$. Then

$$
\begin{aligned}
& G(W)-G\left(W^{o}(\cdot-\widetilde{\varphi})\right) \\
& =G_{W}^{o} \widetilde{W}+\left(G_{W}^{o}(\cdot-\widetilde{\varphi})-G_{W}^{o}\right) \widetilde{W}+ \\
& \int_{0}^{1}(1-s) G_{W W}\left(W^{o}(\cdot-\widetilde{\varphi})+s \widetilde{W}\right) d s[\widetilde{W}, \widetilde{W}] \\
& =G_{W}^{o} \widetilde{W}+\int_{0}^{1}(1-s) G_{W W}\left(W^{o}(\cdot-\widetilde{\varphi})+s \widetilde{W}\right)[\widetilde{W}, \widetilde{W}] \\
& -G_{W W}^{o}(\cdot-s \widetilde{\varphi})\left[W_{x}^{o}(\cdot-s \widetilde{\varphi}) \widetilde{\varphi}, \widetilde{W}\right] d s
\end{aligned}
$$

holds as an equality in $H^{1}$ for almost every $t \in[0, T]$ (see Lemma B.1). The same calculation holds true for $F$ so that $(\widetilde{W}, \widetilde{\varphi}, \widetilde{\lambda})$ satisfies the nonlinear PDAE

$$
\begin{aligned}
\widetilde{W}_{t} & =P \widetilde{W}+\widetilde{\lambda} W_{x}^{o}+\left(\begin{array}{c}
\left(G_{1}+G_{2}\right)_{x}+F_{11}+F_{12} \\
F_{21}+F_{22}
\end{array}\right)+R \\
\widetilde{\varphi}_{t} & =\widetilde{\lambda} \\
0 & =\Psi(\widetilde{W})
\end{aligned}
$$

with initial data

$(3.7 \mathrm{~b})$

$\widetilde{W}(0)=\widetilde{W}_{0}=(\widetilde{u}, \widetilde{v})(0)=\left(\left(W_{0}-W^{o}(\cdot-\widetilde{\varphi}(0))\right), \quad \widetilde{\varphi}(0)=\widetilde{\varphi}_{0}:=\Phi\left(\Psi\left(W_{0}-W^{o}\right)\right)\right.$.

Here $P$ in (3.7a) is given by (1.6). The first equality of (3.7a) holds in $L^{2}\left(\mathbb{R}, \mathbb{R}^{n+m}\right)$ for a.e. $t \in[0, T]$, the other two equalities hold pointwise in $\mathbb{R}$. The nonlinearities 
in $(3.7 \mathrm{a}) \mathrm{read}$

$$
\begin{gathered}
G_{1}=G_{1}(\widetilde{W}, \widetilde{\varphi})=-\int_{0}^{1} D^{2} g\left(W^{o}(\cdot-s \widetilde{\varphi})\right)\left[W_{x}^{o}(\cdot-s \widetilde{\varphi}), \widetilde{\varphi} \widetilde{W}\right] d s, \\
G_{2}=G_{2}(\widetilde{W}, \widetilde{\varphi})=\int_{0}^{1}(1-s) D^{2} g\left(W^{o}(\cdot-\widetilde{\varphi})+s \widetilde{W}\right)[\widetilde{W}, \widetilde{W}] d s, \\
R=R(\widetilde{\varphi}, \widetilde{\lambda})=-\int_{0}^{1} W_{x x}^{o}(\cdot-s \widetilde{\varphi}) d s \widetilde{\varphi} \widetilde{\lambda}
\end{gathered}
$$

and with $g$ replaced by $f_{i}$ for $F_{i j}$. These are quadratic functions of their arguments.

We started the above discussion with a solution of the original PDE (1.3) and obtained a solution of the PDAE reformulation (3.7). Since we also need the other direction, we define the notion of a solution of (3.7).

Definition 3.4. A solution of (3.7) on $[0, T]$ is a quadruple $(\widetilde{u}, \widetilde{v}, \widetilde{\varphi}, \widetilde{\lambda})=$ $(\widetilde{W}, \widetilde{\varphi}, \widetilde{\lambda})$ with

$$
\begin{gathered}
\widetilde{u} \in \mathcal{C}\left([0, T] ; H^{1}\left(\mathbb{R}, \mathbb{R}^{n}\right)\right) \cap H^{1}\left(0, T ; L^{2}\left(\mathbb{R}, \mathbb{R}^{n}\right) \cap L^{2}\left(0, T ; H^{2}\left(\mathbb{R}, \mathbb{R}^{n}\right),\right.\right. \\
\widetilde{v} \in \mathcal{C}\left([0, T] ; H^{1}\left(\mathbb{R}, \mathbb{R}^{m}\right)\right) \cap H^{1}\left(0, T ; L^{2}\left(\mathbb{R}, \mathbb{R}^{m}\right),\right. \\
\widetilde{\varphi} \in \mathcal{C}^{1}([0, T] ; \mathbb{R}), \quad \widetilde{\lambda} \in \mathcal{C}([0, T] ; \mathbb{R}),
\end{gathered}
$$

so that (3.7b) is satisfied, and the first equality in (3.7a) holds in $L^{2}\left(0, T ; \mathbb{R}^{n+m}\right)$ and the last two equalities hold in $\mathbb{R}$ for all $0 \leq t \leq T$. It is called a solution on $\left[0, T^{*}\right)$ with $0<T^{*} \leq \infty$ if it is a solution on $[0, T]$ for all $0<T<T^{*}$.

As usual, initial data for the PDAE cannot be chosen arbitrarily, but are restricted to some manifold by the algebraic condition $0=\Psi(\widetilde{W})$ and the hidden constraint $0=\Psi\left(\widetilde{W}_{t}\right)$. For small $\left(\widetilde{W}_{0}, \widetilde{\varphi}_{0}\right)$ the hidden constraint uniquely determines $\widetilde{\lambda}$.

Now, if $(\widetilde{W}, \widetilde{\varphi}, \widetilde{\lambda})$ is a solution of (3.7) in the sense of Definition 3.4, the discussion that leads to the PDAE can be reversed. We summarize this in the following theorem.

TheOREM 3.5. Let the setting be as above, i.e. $\lambda^{o}=0$ and Assumptions 1.1, 1.2, 3.1 hold.

If $W=(u, v)$ is the unique solution of $(1.3)$ in $[0, T]$ and satisfies

$$
\Psi\left(W-W^{o}\right) \in V \quad \text { for all } t \in[0, T],
$$

then $(\widetilde{W}, \widetilde{\varphi}, \widetilde{\lambda})$ with $(\widetilde{W}, \widetilde{\varphi})=\Upsilon(W)$ and $\widetilde{\lambda}=\widetilde{\varphi}_{t}$, is a solution of (3.7) and

$$
\widetilde{\varphi}(t) \in U \quad \text { for all } t \in[0, T] .
$$

Conversely, if $(\widetilde{W}, \widetilde{\varphi}, \widetilde{\lambda})$ is a solution of (3.7), satisfying (3.10), then $W=\Xi(\widetilde{W}, \widetilde{\varphi})$ solves (1.3) with initial data $W_{0}=\widetilde{W}_{0}+W^{o}\left(\cdot-\widetilde{\varphi}_{0}\right)$ and satisfies (3.9).

Furthermore, the two transitions $\Upsilon$ and $\Xi$ are inverse to each other, i.e.

$$
\left(\begin{array}{c}
\text { PDE-solution } \\
\text { with (3.9) }
\end{array}\right) \stackrel{\Upsilon}{\mapsto}\left(\begin{array}{c}
\text { PDAE-solution } \\
\text { with (3.10) }
\end{array}\right), \quad\left(\begin{array}{c}
\text { PDAE-solution } \\
\text { with (3.10) }
\end{array}\right) \Xi\left(\begin{array}{c}
\text { PDE-solution } \\
\text { with (3.9) }
\end{array}\right) \text {. }
$$

Proof. That solutions $W$ of (1.3) with (3.9) lead to solutions of the PDAE (3.7) has been shown above. For the other direction note that if $(\widetilde{W}, \widetilde{\varphi}, \widetilde{\lambda})$ is a solution of (3.7) with (3.10), then $u=\widetilde{u}+u^{o}(\cdot-\widetilde{\varphi})$ and $v=\widetilde{v}+v^{o}(\cdot-\widetilde{\varphi})$ satisfy the smoothness (2.10). Therefore, the discussion leading to (3.7) can be reversed, 
so that $(u, v)$ is a solution of (1.3) with initial data $u_{0}=\widetilde{u}_{0}+u^{o}\left(\cdot-\widetilde{\varphi}_{0}\right)$ and $v_{0}=\widetilde{v}_{0}+v^{o}\left(\cdot-\widetilde{\varphi}_{0}\right)$.

It remains to show that $\widetilde{W}+W^{o}(\cdot-\widetilde{\varphi})$ satisfies (3.9), but this follows because $\widetilde{W} \in \mathcal{N}(\Psi)$ and $\Psi\left(\widetilde{W}+W^{o}(\cdot-\widetilde{\varphi})-W^{o}\right)=\Psi\left(W^{o}(\cdot-\widetilde{\varphi})-W^{o}\right)=E(\widetilde{\varphi}) \in V$.

\section{Spectral Properties of Linearizations}

We use the Laplace-technique to prove stability for the PDAE reformulation (3.7). The approach of using the Laplace-transform to obtain stability results for time-dependent problems is well-known, see for example $[\mathbf{1 3}, \mathbf{1 5}]$ and references therein. To use the technique for the PDAE reformulation has been first used in $[19,20]$ in the case of traveling waves in hyperbolic PDEs. The method works as follows: Higher order terms of the nonlinear equation are considered as part of the forcing and the equation is considered as linear but inhomogeneous. Global existence and stability follow from local existence of the nonlinear problem plus stability of the linear inhomogeneous problem. Therefore, a major step is the proof of linear stability for inhomogeneous problems.

The linear but inhomogeneous problem for (3.7) has the structure

$$
\begin{aligned}
W_{t} & =P W+\lambda W_{x}^{o}+\left(\begin{array}{c}
G_{x}(x, t)+F_{1}(x, t) \\
F_{2}(x, t)
\end{array}\right), \\
\varphi_{t} & =\lambda, \\
0 & =\Psi(W),
\end{aligned}
$$

with $P$ given by (1.6). To keep the notation simple, we use the same letters as in the nonlinear problem to highlight the terms they are related to in (3.7).

Because the $\varphi$-equation decouples, it suffices to consider the reduced system

$$
\begin{aligned}
W_{t} & =P W+\lambda W_{x}^{o}+\left(\begin{array}{c}
G_{x}(x, t)+F_{1}(x, t) \\
F_{2}(x, t)
\end{array}\right), \\
0 & =\Psi(W) .
\end{aligned}
$$

Assuming $W(0)=0$, application of the Laplace transform, leads to the resolvent equation which we write in operator-matrix form

$$
\mathcal{A}(s)\left(\begin{array}{c}
\widehat{W} \\
\widehat{\lambda}
\end{array}\right):=\left(\begin{array}{cc}
(s I-P) & -W_{x}^{o} \\
\Psi & 0
\end{array}\right)\left(\begin{array}{c}
\widehat{W} \\
\widehat{\lambda}
\end{array}\right)=\left(\left(\begin{array}{c}
\widehat{G}_{x}+\widehat{F}_{1} \\
\widehat{F}_{2} \\
0
\end{array}\right)\right) .
$$

The operator $\mathcal{A}(s)$ acts on $L^{2}\left(\mathbb{R}, \mathbb{C}^{n+m} \times \mathbb{C}\right)$ with domain $H^{2}\left(\mathbb{R}, \mathbb{C}^{n}\right) \times H^{1}\left(\mathbb{R}, \mathbb{C}^{m}\right) \times$ $\mathbb{C}$. Of course, the spectral properties of $\mathcal{A}$ are closely related to the spectral properties of $P$ and we begin with the analysis of the latter. Note that the well-posedness result from Proposition 5.6 below, justifies the use of Laplace transform.

4.1. Spectral Properties of the PDE Operator P. Beside Assumptions 1.1 and 1.3 we impose on the coefficients of $P$ :

Assumption 4.1. (1) The real diagonal matrix $B_{22}$ is invertible,

(2) $B, C \in \mathcal{C}_{b}^{1}\left(\mathbb{R}, \mathbb{C}^{n+m, n+m}\right)$ and their derivatives are asymptotically constant, i.e.

$$
\begin{aligned}
& \exists \lim _{x \rightarrow \pm \infty} B(x)=: B_{ \pm}, \exists \lim _{x \rightarrow \pm \infty} B_{x}(x), \\
& \exists \lim _{x \rightarrow \pm \infty} C(x)=: C_{ \pm}, \exists \lim _{x \rightarrow \pm \infty} C_{x}(x),
\end{aligned}
$$


(3) there is $\delta>0$ so that for all $\omega \in \mathbb{R}, s \in \sigma\left(-\omega^{2} A+i \omega B_{+}+C_{+}\right) \cup \sigma\left(-\omega^{2} A+\right.$ $i \omega B_{-}+C_{-}$) implies $\operatorname{Re} s \leq-\delta<0$.

Recall that 1. is satisfied in the Hodgkin-Huxley case for every traveling wave solution with nonzero speed. In view of Assumption 1.2, linearizations immediately satisfy 2 . We refer to the last assumption as spectral assumption. Consider the resolvent equation

$$
(s I-P)\left(\begin{array}{l}
u \\
v
\end{array}\right)=\left(\begin{array}{c}
\widehat{F}_{1}+\widehat{G}_{x} \\
\widehat{F}_{2}
\end{array}\right) \quad \text { in } L^{2} \times L^{2} .
$$

Using $z=\left(z_{1}, z_{2}, z_{3}\right)^{T}=\left(u, A u_{x}+\widehat{G}, v\right)^{T}$, we rewrite (4.4) as $L(s) z=h$ with

$$
L(s) z=z_{x}-M(x, s) z \quad \text { and } \quad h=\left(\begin{array}{c}
-A_{11}^{-1} \widehat{G} \\
-\widehat{F}_{1}+B_{12} B_{22}^{-1} \widehat{F}_{2}+B_{11} A_{11}^{-1} \widehat{G} \\
-B_{22}^{-1} \widehat{F}_{2}
\end{array}\right),
$$

where the matrix $M(x, s)$ is given by

$$
\left(\begin{array}{ccc}
0 & A_{11}^{-1} & 0 \\
B_{12} B_{22}^{-1} C_{21}+s I-C_{11} & -B_{11} A_{11}^{-1} & -C_{12}-B_{12} B_{22}^{-1}\left(s I-C_{22}\right) \\
-B_{22}^{-1} C_{21} & 0 & B_{22}^{-1}\left(s I-C_{22}\right)
\end{array}\right) .
$$

Let $M_{ \pm}(s):=\lim _{x \rightarrow \infty} M(x, s)$. In [6] we have shown

Lemma 4.2 ([6, Lemma 3.4]). The operator sI $-P$ on $L^{2} \times L^{2}$ with domain $H^{2} \times H^{1}$ is Fredholm if and only if $L(s)$ on $H^{1} \times L^{2} \times L^{2}$ with domain $H^{2} \times H^{1} \times H^{1}$ is Fredholm. In this case their indices coincide and $\operatorname{dim} \mathcal{N}(s I-P)=\operatorname{dim} \mathcal{N}(L(s))$.

Proof. The last assertion is not stated in the original lemma, but is part of its proof.

A result of Palmer [16, Lemma 4.2] relates the Fredholm properties of $L(s)$ to exponential dichotomies and with this to the spectral properties of the limits $M_{ \pm}(s)$, see also [6, Cor. A.5] for a version that directly applies here. For convenience we recall the definition of an exponential dichotomy and several needed properties in Appendix C.

Lemma 4.3. The operator $L(s)$ on $H^{1} \times L^{2} \times L^{2}$ with domain $H^{2} \times H^{1} \times H^{1}$ is Fredholm if and only if the limits $M_{ \pm}(s)$ are hyperbolic. In this case the index of the operator is $\operatorname{ind}(L(s))=\operatorname{dim} E_{+}^{s}(s)-\operatorname{dim} E_{-}^{s}(s)$, where $E_{ \pm}^{s}(s)$ are the stable subspaces of $M_{ \pm}(s)$.

The next lemma shows that the third part of Assumption 4.1 implies that $L(s)$ and therefore also $s I-P$ are Fredholm operators for all $\operatorname{Re} s>-\delta$.

Lemma 4.4 ([6, Lemma 3.2]). For every $s, \kappa \in \mathbb{C}$ holds $s \in \sigma\left(\kappa^{2} A+\kappa B_{ \pm}+C_{ \pm}\right)$ if and only if $\operatorname{det}\left(\kappa I-M_{ \pm}(s)\right)=0$.

Because of Lemma 4.4 and 4.3 the index of $L(s)$ is constant in $\{\operatorname{Re}>-\delta\}$. By Proposition A.2 $\operatorname{dim} E_{+}^{s}(s)=\operatorname{dim} E_{-}^{s}(s)$ for some $s \in \mathbb{R}$, sufficiently large. Using Lemma C.4 this proves

Proposition 4.5. The operator $s I-P$ is Fredholm of index 0 for every $s \in \mathbb{C}$ with $\operatorname{Re} s>-\delta$. 
4.1.1. Resolvent estimates for large $|\mathbf{s}|$. To obtain resolvent estimates for large $|s|$ we consider the "parabolic part" and "hyperbolic part" separately. Resolvent estimates for the parabolic part in this regime can easily be obtained. They are based on the fact that $A_{11}$ is positive definite and, therefore, the essential spectrum lies to the left of a parabola in the left half plane. No further spectral assumption is needed. Solution estimates for this part of the problem, i.e. for

$$
\left(s I_{n}-A_{11} \partial_{x}^{2}-B_{11} \partial_{x}-C_{11}\right) u=f+g_{x} \text { in } L^{2}
$$

are proved in [13]. Since the operator is also Fredholm of index zero (see [4]), holds

LEMma $4.6\left(\left[\mathbf{1 3}\right.\right.$, Lem. 2.1,2.2],[4]). There are $\rho_{1}, K_{1}>0$, so that for all $\left.s \in \mathbb{C}, \operatorname{Re} s>-\delta,|s| \geq \rho_{1}\right\}$, and $f \in L^{2}\left(\mathbb{R}, \mathbb{C}^{n}\right), g \in H^{1}\left(\mathbb{R}, \mathbb{C}^{n}\right),(4.7)$ has a unique solution $u \in H^{2}\left(\mathbb{R}, \mathbb{C}^{n}\right)$. This satisfies

$$
|s|^{2}\|u\|^{2}+|s|\left\|u_{x}\right\|^{2} \leq K_{1}\left(\|f\|^{2}+|s|\|g\|^{2}\right) .
$$

The analysis of the hyperbolic part is more involved. Here the essential spectrum is (asymptotically) parallel to the imaginary axis and it is not possible to obtain uniform estimates without deeper knowledge of its asymptotics. To derive information about the essential spectrum from the Assumption 4.13 , we use the following lemma which is a consequence of a perturbation result from linear algebra. For convenience its proof is given in Appendix A.

Lemma 4.7. Impose Assumption 4.1 3. Then for all $\delta_{0}<\delta$ exists $\omega_{0}$ so that $s \in \sigma\left(i \omega B_{22 \pm}+C_{22 \pm}\right)$ implies $\operatorname{Re} s<-\delta_{0}$ for all $\omega \in \mathbb{R}$ with $|\omega|>\omega_{0}$.

Let $0<\delta_{0}<\delta$ be arbitrary. By Lemma 4.7 there is $\omega_{0}$ so that for $s \in$ $\sigma\left(i \omega B_{22 \pm}+C_{22 \pm}\right)$ with $\omega \in \mathbb{R},|\omega|>\omega_{0}$ follows Re $s<-\delta_{0}$. Therefore, $s \in$ $\sigma\left(i \omega B_{22 \pm}+C_{22 \pm}\right)$, with $|s| \geq \omega_{0} \max \left(\left|B_{22-}\right|_{\infty},\left|B_{22+}\right|_{\infty}\right)+\max \left(\left|C_{22-}\right|_{\infty},\left|C_{22+}\right|_{\infty}\right)$ and $\omega \in \mathbb{R}$, implies $\operatorname{Re} s<-\delta_{0}$. This discussion shows that [19, Prop. 3.8] applies to the hyperbolic part of our problem:

Lemma 4.8 ([19, Prop. 3.8]). Let $0<\delta_{0}<\delta$ be arbitrary. Then there are $\rho_{0}, K_{0}>0$ so that for all $\operatorname{Re} s>-\delta_{0},|s| \geq \rho_{0}$, and every $f \in H^{1}\left(\mathbb{R}, \mathbb{C}^{m}\right)$, the resolvent equation

$$
\left(s I_{m}-B_{22} \partial_{x}-C_{22}\right) v=f
$$

has a unique solution $v \in H^{1}$ and

$$
\|v\|_{L^{2}}^{2} \leq K_{0}\|f\|_{L^{2}}^{2}, \quad\|v\|_{H^{1}}^{2} \leq K_{0}\|f\|_{H^{1}}^{2} .
$$

For the coupled problem let $(u, v)^{T}$ be a solution of (4.4), i.e.

$$
\begin{aligned}
\left(s I_{n}-A_{11} \partial_{x}^{2}-B_{11} \partial_{x}-C_{11}\right) u & =\left(\widehat{F}_{1}+C_{12} v-B_{12, x} v\right)+\left(\widehat{G}+B_{12} v\right)_{x}, \\
\left(s I_{m}-B_{22} \partial_{x}-C_{22}\right) v & =\widehat{F}_{2}+C_{21} u,
\end{aligned}
$$

so that by (4.10) and (4.8) follow

$$
\begin{gathered}
\|v\|^{2} \leq K_{0}\left\|\widehat{F}_{2}+C_{21} u\right\|^{2} \leq c\left(\left\|\widehat{F}_{2}\right\|^{2}+\|u\|^{2}\right), \text { and } \\
|s|^{2}\|u\|^{2}+|s|\left\|u_{x}\right\|^{2} \leq K_{1}\left(\left\|\widehat{F}_{1}+C_{12} v-B_{12, x} v\right\|^{2}+|s|\left\|\widehat{G}+B_{12} v\right\|^{2}\right) \\
\leq c\left(\left\|\widehat{F}_{1}\right\|^{2}+|s|\|\widehat{G}\|^{2}+|s|\left\|\widehat{F}_{2}\right\|^{2}+|s|\|u\|^{2}\right) .
\end{gathered}
$$


Therefore, for sufficiently large $|s|$ with $\operatorname{Re} s>-\delta_{0}$ we can bring the $u$ - and $v$-terms to the left hand side and using the $H^{1}$-estimate from Lemma 4.8 yields

$$
|s|\|u\|^{2}+\left\|u_{x}\right\|^{2}+\|v\|_{H^{1}}^{2} \leq K\left(\frac{1}{|s|}\left\|\widehat{F}_{1}\right\|^{2}+\|\widehat{G}\|^{2}+\left\|\widehat{F}_{2}\right\|_{H^{1}}^{2}\right) .
$$

Because of Fredholm's alternative, Proposition 4.5, this also implies solvability:

Proposition 4.9. Let $0<\delta_{0}<\delta$ be arbitrary. Then there are $\rho, K>0$ so that for all $s \in\left\{s \in \mathbb{C}: \operatorname{Re} s>-\delta_{0},|s| \geq \rho\right\}$ and all right hand sides $\widehat{F}_{1} \in L^{2}$, $\widehat{G} \in H^{1}, \widehat{F}_{2} \in H^{1}$ there is a unique solution $W=(u, v)^{T} \in H^{2} \times H^{1}$ of (4.4). The solution satisfies (4.11).

4.1.2. Resolvent estimates for bounded $|\mathbf{s}|$. Assume $s_{0} \in \rho(P), \rho(P)$ the resolvent set of $P$. Then there is no nontrivial bounded solution of $\left(s_{0} I-P\right) W=0$ and by Lemma 4.2 there is no nontrivial bounded solution of $L\left(s_{0}\right) z=0$, which implies that $L\left(s_{0}\right)$ has an exponential dichotomy (ED) on the whole real line, see [7]. The Roughness Theorem C.3 shows that the exponent $\beta$ and constant $K$ of the dichotomy data can locally be chosen independently of $s$. Therefore, it is possible to chose the same exponent and constant for the dichotomy data for all $s$ from a compact subset of $\rho(P)$.

Proposition 4.10. Let $\Omega \subset\{\operatorname{Re} s>-\delta\} \cap \rho(P)$ be compact. Then there is $K>0$ so that for all $s \in \Omega$ and all right hand sides $\widehat{F}_{1} \in L^{2}, \widehat{G} \in H^{1}, \widehat{F}_{2} \in H^{1}$ there is a unique solution $W=(u, v)^{T} \in H^{2} \times H^{1}$ of (4.4). The solution satisfies

$$
\|u\|_{H^{1}}^{2}+\|v\|_{H^{1}}^{2} \leq K\left(\left\|\widehat{F}_{1}\right\|^{2}+\|\widehat{G}\|^{2}+\left\|\widehat{F}_{2}\right\|^{2}\right) .
$$

Proof. Let $s \in \Omega$ be arbitrary and rewrite the problem (4.3) with $z=$ $\left(u, A u_{x}+\widehat{G}, v\right)^{T}$ as the first order equation $L(s) z=h(4.5)$. This operator has an exponential dichotomy on $\mathbb{R}$ with data $(K, \beta, \pi(s))$, where $K$ and $\beta$ can be chosen independently of $s \in \Omega$. Theorem C.2 proves unique solvability of $L(s) z=h$ and the estimate $\|z\|^{2} \leq 5 K^{2} \beta^{-2}\|h\|^{2}$. With the differential equation follows $\|z\|_{H^{1}}^{2} \leq c\|h\|^{2}$, where $C$ does not depend on $s \in \Omega$. Recalling the definition of $z$ and (4.5), one obtains $\|u\|_{H^{1}}^{2}+\|v\|_{H^{1}}^{2} \leq c\|z\|_{H^{1}}^{2}$ and $\|h\|^{2} \leq c\left(\|\widehat{G}\|^{2}+\left\|\widehat{F}_{1}\right\|^{2}+\left\|\widehat{F}_{2}\right\|^{2}\right)$. Combination proves the assertion.

4.2. Spectral Properties of the PDAE Operator $\mathcal{A}$. The estimates for $s I-P$ are now used to derive solution estimates for (4.3). First we note that Proposition 4.5 implies together with the bordering Lemma [3, Lem. 2.3]:

Proposition 4.11. The operator $\mathcal{A}(s): H^{2} \times H^{1} \times \mathbb{C} \rightarrow L^{2} \times L^{2} \times \mathbb{C}$ is Fredholm of index 0 for every $s \in \mathbb{C}$ with $\operatorname{Re} s>-\delta$.

It also proves useful to define the linear projector $\Pi$ on $L^{2} \times L^{2}$,

$$
\Pi:(u, v)^{T} \mapsto\left(\begin{array}{c}
u_{x}^{o} \\
v_{x}^{o}
\end{array}\right) \frac{\Psi(u, v)}{\Psi\left(u_{x}^{o}, v_{x}^{o}\right)} .
$$

This operator projects $(u, v)^{T} \in L^{2} \times L^{2}$ along $\mathcal{N}(\Psi)$ onto $\mathcal{N}(P)=\left\{c\left(u_{x}^{o}, v_{x}^{o}\right)^{T}: c \in\right.$ $\mathbb{C}\}$. The following lemma is a simple consequence of the assumptions on $\left(u_{x}^{o}, v_{x}^{o}\right)$ and on $\Psi$.

LEMMA 4.12. The linear operator $\Pi: L^{2} \times L^{2} \rightarrow L^{2} \times L^{2}$ is bounded and continuously extends to $\Pi: H^{-1} \times H^{-1} \rightarrow H^{2} \times H^{2}$. In particular, the mapping $\Pi \circ P: H^{2} \times H^{1} \rightarrow H^{2} \times H^{2}$ has a continuous extension to $\Pi \circ P: H^{1} \times L^{2} \rightarrow H^{2} \times H^{2}$. 
4.2.1. Large $|\mathbf{s}|$. For large absolute values of $s$ in a right half plane we have the following resolvent estimate.

Proposition 4.13. For $0<\delta_{0}<\delta$ exist $C_{0}, K_{L}>0$ so that for all $s \in \mathbb{C}$, $\operatorname{Re} s \geq-\delta_{0},|s|>C_{0}$ and all right hand sides $\widehat{F}_{1} \in L^{2}\left(\mathbb{R}, \mathbb{C}^{n}\right), \widehat{G} \in H^{1}\left(\mathbb{R}, \mathbb{C}^{n}\right), \widehat{F}_{2} \in$ $H^{1}\left(\mathbb{R}, \mathbb{C}^{m}\right)$, exists a unique solution $(\widehat{W}, \widehat{\lambda})$ of $(4.3)$. It holds $\widehat{W} \in H^{2}\left(\mathbb{R}, \mathbb{C}^{n}\right) \times$ $H^{1}\left(\mathbb{R}, \mathbb{C}^{m}\right)$ and

$$
\|\widehat{W}\|_{H^{1}}+|\widehat{\lambda}| \leq K_{L}\left(\left\|\widehat{F}_{1}\right\|_{L^{2}}+\|\widehat{G}\|_{L^{2}}+\left\|\widehat{F}_{2}\right\|_{H^{1}}\right) .
$$

IDEA OF PROOF. By Lemma 4.9 there are $K, C_{0}>0$ so that for all $s \in \mathbb{C}$ there exists a unique solution $w_{0}=\left(u_{0}, v_{0}\right)^{T} \in H^{2}(\mathbb{R}) \times H^{1}(\mathbb{R})$ of

$$
(s I-P) w=\left(\begin{array}{c}
\widehat{F}_{1}+\widehat{G}_{x} \\
\widehat{F}_{2}
\end{array}\right) \quad \text { in } L^{2}(\mathbb{R}) \times L^{2}(\mathbb{R}) .
$$

This satisfies the estimate (4.11). Then $\widehat{W}:=(I-\Pi) w_{0}$ and $\widehat{\lambda}:=-s \frac{\Psi\left(w_{0}\right)}{\Psi\left(w_{x}^{o}\right)}$ solve (4.3) and satisfy estimate (4.14).

For the details we refer to $[\mathbf{1 9}$, Lem. 4.3$]$, which easily adapts to the current situation.

4.2.2. Compact subsets of the resolvent set. For $s$ from a compact set $\Omega \subset\{s \in$ $\mathbb{C}: \operatorname{Re} s>-\delta, s \neq 0\}$ we generalize (4.3) to

$$
\mathcal{A}(s)\left(\begin{array}{c}
\widehat{W} \\
\widehat{\lambda}
\end{array}\right)=\left(\left(\begin{array}{c}
\widehat{G}_{x}+\widehat{F}_{1} \\
\widehat{F}_{2} \\
\sigma
\end{array}\right),\right.
$$

where $\sigma \in \mathbb{C}$ is arbitrary. In compact regions we have

Proposition 4.14. For every $\Omega$ as above there is a constant $K_{C}>0$ so that for all $s \in \Omega$ and all right hand sides $\widehat{F}_{1} \in L^{2}\left(\mathbb{R}, \mathbb{C}^{n}\right), \widehat{G} \in H^{1}\left(\mathbb{R}, \mathbb{C}^{n}\right), \widehat{F}_{2} \in H^{1}\left(\mathbb{R}, \mathbb{C}^{m}\right)$, $\sigma \in \mathbb{C}$, there exists a unique solution $(\widehat{W}, \widehat{\lambda})$ of $(4.15)$. It holds $\widehat{W} \in H^{2}\left(\mathbb{R}, \mathbb{C}^{n}\right) \times$ $H^{1}\left(\mathbb{R}, \mathbb{C}^{m}\right)$ and

$$
\|\widehat{W}\|_{H^{1}}+|\widehat{\lambda}| \leq K_{C}\left(\left\|\widehat{F}_{1}\right\|_{L^{2}}+\|\widehat{G}\|_{L^{2}}+\left\|\widehat{F}_{2}\right\|_{L^{2}}\right) .
$$

We do not give the proof because it is basically the same as that of Proposition 4.13, but this time define $\widehat{W}:=(I-\Pi) w^{0}+\sigma \frac{W_{x}^{o}}{\Psi\left(W_{x}^{o}\right)}$ and $\widehat{\lambda}:=-s \frac{\Psi\left(w^{0}\right)-\sigma}{\Psi\left(W_{x}^{o}\right)}$.

4.2.3. Small $|\mathbf{s}|$. Finally, we prove solution estimates for (4.3) in a small neighborhood of zero. Here the algebraic constraint is crucial because it removes zero from the spectrum of the operator.

Proposition 4.15. There are $c_{0}, K_{S}>0$ so that for all $s \in \mathbb{C}$ with $|s|<c_{0}$ and all right hand sides $\widehat{F}_{1} \in L^{2}(\mathbb{R}), \widehat{G} \in H^{1}(\mathbb{R})$, and $\widehat{F}_{2} \in L^{2}(\mathbb{R})$ there is a unique solution $(\widehat{W}, \widehat{\lambda}), \widehat{W}=(\widehat{u}, \widehat{v}) \in H^{2} \times H^{1}$, of (4.3) and

$$
\|\widehat{u}\|_{H^{2}}+\|\widehat{v}\|_{H^{1}}+|\widehat{\lambda}| \leq K_{S}\left(\left\|\widehat{F_{1}}\right\|+\|\widehat{G}\|_{H^{1}}+\left\|\widehat{F_{2}}\right\|\right) .
$$

Proposition 4.15 easily follows with a perturbation argument from the next lemma. 
Lemma 4.16. There is $c>0$ so that for every $\widehat{F}_{1} \in L^{2}(\mathbb{R}), \widehat{G} \in H^{1}(\mathbb{R})$, $\widehat{F}_{2} \in L^{2}(\mathbb{R})$, and $\sigma \in \mathbb{C}$ there is a unique solution $(w, \lambda), w=(u, v) \in H^{2} \times H^{1}$, of (4.15) and

$$
\|u\|_{H^{2}}+\|v\|_{H^{1}}+|\lambda| \leq c\left(\left\|\widehat{F}_{1}\right\|+\|\widehat{G}\|_{H^{1}}+\left\|\widehat{F}_{2}\right\|+|\sigma|\right) .
$$

Proof. Consider $\mathcal{A}(0)$ as a bounded linear operator from $H^{2} \times H^{1} \times \mathbb{C}$ to $L^{2} \times L^{2} \times \mathbb{C}$. Assume that $H^{2} \times H^{1} \times \mathbb{C} \ni(w, \lambda)^{T}=(u, v, \lambda)^{T} \in \mathcal{N}(\mathcal{A}(0))$. This implies

$$
P\left(\begin{array}{l}
u \\
v
\end{array}\right)=-\lambda\left(\begin{array}{l}
u_{x}^{o} \\
v_{x}^{o}
\end{array}\right)
$$

which is only possible if $\lambda=0$ since 0 is a simple eigenvalue of $P$ by Assumption 1.3. Therefore, $(u, v) \in \mathcal{N}(P)$, i.e. $(u, v)=\nu\left(u_{x}^{o}, v_{x}^{o}\right)$ for some $\nu \in \mathbb{C}$. But $\mathcal{A}(0)\left(\nu u_{x}^{o}, \nu v_{x}^{o}, 0\right)^{T}=0$ implies $\nu \Psi\left(u_{x}^{o}, v_{x}^{o}\right)=0$, what is equivalent to $\nu=0$ because of Assumption 3.1.

Moreover, by Proposition 4.11, $\mathcal{A}(0)$ is Fredholm of index 0 , what implies that $\mathcal{A}(0): H^{2} \times H^{1} \times \mathbb{C} \rightarrow L^{2} \times L^{2} \times \mathbb{C}$ is a linear homeomorphism. Therefore, the solution $(u, v, \lambda)$ of $(4.15)$ satisfies for some constant $c$ the estimate

$$
\|u\|_{H^{2}}+\|v\|_{H^{1}}+|\lambda| \leq \operatorname{const}\left(\left\|\widehat{F}_{1}\right\|_{L^{2}}+\left\|\widehat{G}_{x}\right\|_{L^{2}}+\left\|\widehat{F}_{2}\right\|_{L^{2}}+|\sigma|\right) .
$$

Proof of Proposition 4.15. Lemma 4.16 shows that $\mathcal{A}(0): H^{2} \times H^{1} \times \mathbb{C} \rightarrow$ $L^{2} \times L^{2} \times \mathbb{C}$ is a linear homeomorphism. Then for small $|s|$ the assertion follows from a simple perturbation argument.

With a von Neumann series argument we also obtain analytic dependence of $\mathcal{A}(s)^{-1}$ on $s$ from the results of Lemma 4.16 and Proposition 4.14:

Corollary 4.17. For every $s \in \mathbb{C}$ with $\operatorname{Re} s>-\delta$ the operator $\mathcal{A}(s)$ from (4.3) is a linear homeomorphism and its inverse $\mathcal{A}(s)^{-1}$ depends holomorphically on $s$ for $\operatorname{Re} s>-\delta$.

\section{PDAE Stability}

Now we prove stability for the PDAE reformulation (3.7). We begin with linear stability (Theorem 5.1) and then use the linear result to prove nonlinear stability (Theorem 5.9). Without mentioning it again, we always impose Assumptions 1.1, 1.2 , and 3.1. We again denote by $W$ the vector $(u, v)^{T}$, consisting of the functions $u$ and $v$, corresponding to the "parabolic part" and "hyperbolic part", respectively.

5.1. Linear PDAE Stability. We begin with the analysis of (4.2). First we show exponential well-posedness of the linear PDAE problem to justify application of the Laplace transform. In the second step we use the resolvent estimates from Section 4 to deduce linear stability. The linear result is the following theorem. The precise meaning of a solution is given in Definition 5.2 below.

TheOREm 5.1 (Linear PDAE Stability). For all $F_{1}, F_{2}, G \in L_{l o c}^{\infty}\left(J ; H^{1}\right), J=$ $[0, T]$ with $T>0$ or $J=[0, \infty)$, and all consistent initial data $W_{0}=\left(u_{0}, v_{0}\right)^{T} \in$ $H^{2}\left(\mathbb{R}, \mathbb{R}^{n+m}\right), \lambda_{0} \in \mathbb{R}$, there is a unique weak solution $(W, \lambda)^{T}=(u, v, \lambda)^{T}$ of $(4.2)$ in $J$. 
The solution is a strong solution. Moreover, for every $\eta_{0}<\delta$, $\delta$ from Assumptions 1.3 and 4.1 , exists $C_{l}$, independent of $F_{1}, F_{2}, G, u_{0}, v_{0}, \lambda_{0}$, so that for all $\eta \leq \eta_{0}$ and all $t \in J$,

$$
\begin{aligned}
& \|W(t)\|_{H^{1}}^{2}+e^{-2 \eta t} \int_{0}^{t} e^{2 \eta \tau}\left\{\|W(\tau)\|_{H^{1}}^{2}+|\lambda(\tau)|^{2}\right\} d \tau \\
& \leq C_{l} e^{-2 \eta t}\left[\left\|W_{0}\right\|_{H^{2}}^{2}+\int_{0}^{t} e^{2 \eta \tau}\left\{\|G(\tau)\|_{H^{1}}^{2}+\left\|F_{1}(\tau)\right\|_{L^{2}}^{2}+\left\|F_{2}(\tau)\right\|_{H^{1}}^{2}\right\} d \tau\right] .
\end{aligned}
$$

Finally, if $F_{1}, F_{2}, G \in \mathcal{C}\left(J ; L^{2}\right)$, then also $\lambda \in \mathcal{C}([0, \infty))$.

5.1.1. Exponential Well-Posedness of the PDAE. Consider (4.2) subject to consistent initial data

$$
W(0)=(u(0), v(0))^{T}=\left(u_{0}, v_{0}\right)^{T} \in H^{1} \times H^{1}, \quad \lambda(0)=\lambda_{0} \in \mathbb{R}
$$

i.e. $\Psi\left(u_{0}, v_{0}\right)=0$ and the hidden constraint $\left.\Psi\left(u_{t}, v_{t}\right)\right|_{t=0}=0$ is satisfied. This determines $\lambda_{0}$ in terms of $u_{0}, v_{0}$. For the inhomogeneities of (4.2) we assume

$$
G \in L_{l o c}^{2}\left([0, \infty) ; H^{1}\right), \quad F_{1} \in L_{l o c}^{2}\left([0, \infty) ; H^{1}\right), \quad F_{2} \in L_{l o c}^{2}\left([0, \infty) ; H^{1}\right) .
$$

Definition 5.2. The triple $(u, v, \lambda)$ is called a weak solution of (4.2), (5.2) in $[0, T]$ if

$$
\begin{aligned}
& u \in L^{2}\left(0, T ; H^{1}\right) \cap L^{\infty}\left(0, T ; H^{1}\right) \cap H^{1}\left(0, T ;\left(H^{1}\right)^{\prime}\right), \\
& v \in L^{2}\left(0, T ; H^{1}\right) \cap L^{\infty}\left(0, T ; H^{1}\right) \cap H^{1}\left(0, T ; L^{2}\right), \\
& \lambda \in L^{2}(0, T ; \mathbb{R}),
\end{aligned}
$$

such that $(u, v)$ is a weak solution of the PDE part in the sense of Definition 2.3 and $0=\Psi(u, v)$ holds for a.e. $t \in[0, T]$.

$A$ strong solution in $[0, T]$ is a weak solution $(u, v, \lambda)$ in $[0, T]$ with

$u \in \mathcal{C}\left([0, T] ; H^{1}\right) \cap L^{2}\left(0, T ; H^{2}\right) \cap H^{1}\left(0, T ; L^{2}\right) \quad$ and $\quad v \in \mathcal{C}\left([0, T] ; H^{1}\right) \cap H^{1}\left(0, T ; L^{2}\right)$, so that (4.2) holds in $L^{2} \times L^{2} \times \mathbb{R}$ for almost every $t \in[0, T]$.

The triple $(u, v, \lambda)$ is a weak (respectively strong) solution of the PDAE in $\left[0, T^{*}\right), T^{*} \in(0, \infty]$, if it is a weak (respectively strong) solution in $[0, T]$ for all $0<T<T^{*}$.

For the proof of well-posedness, we project the differential equation part of (4.2) onto the manifold given by the algebraic constraint. The resulting linear PDE problem is exponentially well-posed, see Proposition 5.4. Its solution leads to a solution of the original linear PDAE (4.2). We also obtain exponential wellposedness for (4.2).

Applying the projector $(I-\Pi), \Pi$ from (4.13), to the PDE part of (4.2) leads to

$$
W_{t}=(I-\Pi) P W+(I-\Pi)\left(\begin{array}{c}
F_{1}+G_{x} \\
F_{2}
\end{array}\right), \quad t \geq 0,
$$

subject to $W(0)=W_{0}=\left(u_{0}, v_{0}\right)^{T}=(I-\Pi)\left(u_{0}, v_{0}\right)^{T} \in \mathcal{N}(\Psi)$.

Use $\Pi_{1}=u_{x}^{o} \Psi\left(W_{x}^{o}\right)^{-1} \Psi(\cdot)$ and $\Pi_{2}=v_{x}^{o} \Psi\left(W_{x}^{o}\right)^{-1} \Psi(\cdot)$ to define the operators

$$
\widetilde{g}: W \mapsto D g^{o} W+G,
$$

$$
\begin{aligned}
& \tilde{f}_{1}: W \mapsto D f_{1}^{o} W-\Pi_{1} P W+F_{1}-\Pi_{1}\left(G_{x}+F_{1}\right), \\
& \tilde{f}_{2}: W \mapsto D f_{2}^{o} W-\Pi_{2} P W+F_{2}-\Pi_{2}\left(F_{2}\right) .
\end{aligned}
$$


Then equation (5.4) can be written in the form analyzed in Theorem 2.5:

$$
\left(\begin{array}{l}
u \\
v
\end{array}\right)_{t}=\left(\begin{array}{c}
A_{11} u_{x x}+\widetilde{g}(u, v)_{x}+\widetilde{f}_{1}(u, v) \\
B_{22} v_{x}+\widetilde{f}_{2}(u, v)
\end{array}\right) .
$$

Lemma 5.3. If $F_{1}, G, F_{2}$ satisfy (5.3), the operators $\widetilde{g}, \widetilde{f}_{1}, \widetilde{f}_{2}$ satisfy Assumption 2.1 .

Proof. By Assumption 1.2, $D g^{o}, D f_{1}^{o}$, and $D f_{2}^{o}$ are elements of $\mathcal{C}_{b}^{1}$. Therefore, $\widetilde{g}$ satisfies the properties from Assumption 2.1 because of (5.3) and Lemma 2.2 2 .

For the analysis of $\widetilde{f}_{1}$ and $\widetilde{f}_{2}$ note that $\Pi_{1}$ and $\Pi_{2}$ can be considered as bounded linear operators from $L^{2}\left(t_{0}, T ; H^{-1}\right) \times L^{2}\left(t_{0}, T ; H^{-1}\right)$ into $L^{2}\left(t_{0}, T ; H^{1}\right)$ for $0 \leq$ $t_{0}<T$. By Lemma 4.12, their norm is bounded by a constant $C_{\Pi}$ independent of $0 \leq t_{0}<T$. Thus, $F_{1}-\Pi_{1}\left(G_{x}+F_{1}\right) \in L_{l o c}^{2}\left([0, \infty) ; H^{1}\right)$ and $F_{2}-\Pi_{2}\left(F_{2}\right) \in$ $L_{l o c}^{2}\left([0, \infty) ; H^{1}\right)$.

By Lemma 4.12, also the operators $\Pi_{i} \circ P: L^{2}\left(t_{0}, T ; H^{1}\right) \times L^{2}\left(t_{0}, T: H^{1}\right) \rightarrow$ $L^{2}\left(t_{0}, T ; H^{1}\right), i=1,2$, are bounded by a constant $C_{\Pi P}$, independent of $t_{0}$ and $T$. This and $D f_{1}^{o}, D f_{2}^{o} \in \mathcal{C}_{b}^{1}$ imply that $\tilde{f}_{i}, i=1,2$, maps $L^{2}\left(t_{0}, T ; H^{1}\right) \cap L^{\infty}\left(t_{0}, T ; H^{1}\right)$ into $L^{2}\left(t_{0}, T ; H^{1}\right)$. Moreover, for $i=1,2$, and all

$$
W, W^{\prime} \in L^{2}\left(t_{0}, T ; H^{1}\right) \cap L^{\infty}\left(t_{0}, T ; H^{1}\right),
$$

hold

$$
\left\|\tilde{f}_{i}(W)-\tilde{f}_{i}\left(W^{\prime}\right)\right\|_{L^{2}\left(t_{0}, T ; H^{1}\right)} \leq\left(\left\|D f_{1}^{o}\right\|_{W^{1, \infty}}+C_{\Pi P}\right)\left\|W-W^{\prime}\right\|_{L^{2}\left(t_{0}, T ; H^{1}\right)} .
$$

Now exponential well-posedness of the projected PDE (5.4) is an easy consequence of Theorem 2.5. Here we greatly benefit from the abstract formulation of Theorem 2.5.

Proposition 5.4 (Well-posedness of the projected Cauchy problem). For all $F_{1}, G, F_{2}$ of the form (5.3), the Cauchy problem for (5.4) with $u_{0}, v_{0} \in H^{1}, \Pi\left(u_{0}, v_{0}\right)=$ 0 , has a unique weak solution $(u, v)^{T}$ on $[0, \infty)$, this even is a strong solution. Moreover, for every $\eta_{0} \geq 0$ exists $C$, so that for all $\eta \leq \eta_{0}$ and all $t \geq 0$ holds

$$
\begin{aligned}
\|u(t)\|_{H^{1}}^{2}+\|v(t)\|_{H^{1}}^{2} \leq C e^{C t}\left(e^{\max (-2 \eta t, 0)}\left(\left\|u_{0}\right\|_{H^{1}}^{2}+\left\|v_{0}\right\|_{H^{1}}^{2}\right)\right. \\
\left.\quad+e^{-2 \eta t} \int_{0}^{t} e^{2 \eta \tau}\left\{\|G(\tau)\|_{H^{1}}^{2}+\left\|F_{1}(\tau)\right\|_{L^{2}}^{2}+\left\|F_{2}(\tau)\right\|_{H^{1}}^{2}\right\} d \tau\right) .
\end{aligned}
$$

REMARK 5.5. If there is $\eta \in \mathbb{R}$ so that the integral term in (5.7) is uniformly bounded for all $t \geq 0$, then also the solution is exponentially bounded.

Proof. Because of Lemma 5.3, Theorem 2.5 applies and shows the existence and uniqueness part. To prove (5.7) rewrite (5.4) as

$$
W_{t}=P W+\left[-\Pi P W+(I-\Pi)\left(\begin{array}{c}
G_{x}+F_{1} \\
F_{2}
\end{array}\right)\right], \quad W(0)=W_{0} .
$$

The term $-\Pi P W$ satisfies $\|\Pi P W\|_{L^{2}\left(0, T ; H^{1}\right)} \leq C\|W\|_{L^{2}\left(0, T ; H^{1}\right)}$, for some $C>0$, because of Lemma 4.12, and we consider it as part of the forcing. Then the energy 
estimate, Lemma 2.6, yields for every $\eta_{0} \geq 0$ : There is $C>0$ so that for all $\eta \leq \eta_{0}$ holds

$$
\begin{aligned}
e^{2 \eta t}\|W(t)\|_{H^{1}}^{2} & \leq e^{2 \eta t}\left\|W_{0}\right\|_{H^{1}}^{2}+C \int_{0}^{t} e^{2 \eta \tau}\|W(\tau)\|_{H^{1}}^{2} d \tau \\
& +C \int_{0}^{t} e^{2 \eta \tau}\left(\|G(\tau)\|_{H^{1}}^{2}+\left\|F_{1}(\tau)\right\|_{L^{2}}^{2}+\left\|F_{2}(\tau)\right\|_{H^{1}}^{2}\right) d \tau, \quad \forall t \geq 0 .
\end{aligned}
$$

With Gronwall's inequality [12, Lemma 6.3.6], applied to $e^{2 \eta t}\|W(t)\|_{H^{1}}^{2}$, follows for all $t \geq 0$

$$
\begin{aligned}
e^{2 \eta t}\|W(t)\|_{H^{1}}^{2} \leq C e^{C t} & \left(e^{\max (2 \eta t, 0)}\left\|W_{0}\right\|_{H^{1}}^{2}\right. \\
& \left.+\int_{0}^{t} e^{2 \eta \tau}\left\{\|G(\tau)\|_{H^{1}}^{2}+\left\|F_{1}(\tau)\right\|_{L^{2}}^{2}+\left\|F_{2}(\tau)\right\|_{H^{1}}^{2}\right\} d \tau\right) .
\end{aligned}
$$

This implies (5.7) and finishes the proof.

Well-posedness of the projected equation leads to well-posedness of the linear PDAE:

Proposition 5.6 (Well-posedness of the linear PDAE). For all $F_{1}, G, F_{2}$, satisfying (5.3), and all consistent initial data $u_{0} \in H^{1}, v_{0} \in H^{1}, \lambda_{0} \in \mathbb{R}$, there is a unique weak solution $(u, v, \lambda)$ of $(4.2)$ on $[0, \infty)$, which in fact is a strong solution. Furthermore, if

$$
\int_{0}^{\infty} e^{2 \eta_{L} t}\left(\left\|F_{1}(t)\right\|_{L^{2}}^{2}+\|G(t)\|_{H^{1}}^{2}+\left\|F_{2}(t)\right\|_{H^{1}}^{2}\right) d t<\infty
$$

for some $\eta_{L} \in \mathbb{R}$, there are $k, c \geq 0$, so that

(5.9) $\|u(t)\|_{H^{1}}^{2}+\|v(t)\|_{H^{1}}^{2} \leq k e^{c t}$, for all $t \geq 0, \quad$ and $\quad \int_{0}^{\infty} e^{-c \tau}|\lambda(\tau)|^{2} d \tau<\infty$.

Finally, if there are $\eta_{e}, K_{e} \in \mathbb{R}$ so that $\left(\left\|F_{1}(t)\right\|_{L^{2}}^{2}+\|G(t)\|_{L^{2}}^{2}+\left\|F_{2}(t)\right\|_{L^{2}}^{2}\right) \leq$ $K_{e} e^{-\eta_{e} t}$ for a.e. $t \geq 0$, then also $\lambda$ satisfies $|\lambda(t)|^{2} \leq k e^{\text {ct }}$, for a.e. $t \geq 0$, for some constants $k$ and $c$.

Proof. Step 1: [Uniqueness] Let $(u, v, \lambda)^{T}$ be a weak solution of (4.2) in $[0, T]$. By definition, $W=(u, v)^{T}$ is a weak solution in the sense of Definition 2.3 in $[0, T]$ of

$$
\left(\begin{array}{l}
u \\
v
\end{array}\right)_{t}=P\left(\begin{array}{l}
u \\
v
\end{array}\right)+\left(\begin{array}{c}
G_{x}+F_{1} \\
F_{2}
\end{array}\right)+\lambda W_{x}^{o}, \quad u(0)=u_{0}, \quad v(0)=v_{0} .
$$

The assumptions imply, $G, F_{1}+\lambda u_{x}^{o}, F_{2}+\lambda v_{x}^{o} \in L^{2}\left([0, T] ; H^{1}\right)$, so that $(u, v)^{T}$ is the unique strong solution of (5.10) by Corollary 2.7. In particular,

$$
\begin{aligned}
& u \in \mathcal{C}\left([0, T] ; H^{1}\right) \cap L^{2}\left(0, T ; H^{2}\right) \cap H^{1}\left(0, T ; L^{2}\right), \\
& v \in \mathcal{C}\left([0, T] ; H^{1}\right) \cap H^{1}\left(0, T ; L^{2}\right),
\end{aligned}
$$

and every weak solution of (4.2) already is a strong solution.

By assumption, $\Psi(W)=0$ for a.e. $t \in[0, T]$. Moreover, $t \mapsto \Psi(W(t)) \in$ $H^{1}([0, T])$ by $(5.11)$. As in the proof of Lemma 3.3 its distributional derivative is

$$
\frac{d}{d t} \Psi\left(\begin{array}{l}
u \\
v
\end{array}\right)=\Psi\left(P\left(\begin{array}{l}
u \\
v
\end{array}\right)+\left(\begin{array}{c}
F_{1}+G_{1, x} \\
F_{2}
\end{array}\right)\right)+\lambda \Psi\left(W_{x}^{o}\right)=0, \quad \text { for a.e. } t \in[0, T] .
$$


This equality can be solved for $\lambda$,

$$
\lambda=-\Psi\left(W_{x}^{o}\right)^{-1} \Psi\left(P\left(\begin{array}{l}
u \\
v
\end{array}\right)+\left(\begin{array}{c}
F_{1}+G_{x} \\
F_{2}
\end{array}\right)\right), \quad \text { for a.e. } t \in[0, T] .
$$

Inserting (5.12) into (5.10) and recalling the definition (4.13) of $\Pi$ shows

$$
\left(\begin{array}{l}
u \\
v
\end{array}\right)_{t}=(I-\Pi) P\left(\begin{array}{l}
u \\
v
\end{array}\right)+(I-\Pi)\left(\begin{array}{c}
F_{1}+G_{x} \\
F_{2}
\end{array}\right),
$$

where the equality holds in $L^{2}\left(\mathbb{R}, \mathbb{R}^{p}\right) \times L^{2}\left(\mathbb{R}, \mathbb{R}^{m}\right)$ for a.e. $t \in[0, T]$. Therefore, $(u, v)^{T}$ solves (5.4) and is uniquely determined by Proposition 5.4.

Step 2: [Existence] Let $W:=(u, v)^{T}$ solve (5.4) and define $\lambda$ by (5.12). By Proposition $5.4 u$ and $v$ satisfy (5.11). Moreover, because of Lemma 4.12, for a.e. $t \in[0, \infty)$,

(5.13) $|\Psi(P W)|+\left|\Psi\left(\left(G_{x}+F_{1}, F_{2}\right)^{T}\right)\right| \leq c\left(\|W\|_{H^{1}}+\left\|F_{1}\right\|_{L^{2}}+\|G\|_{L^{2}}+\left\|F_{2}\right\|_{L^{2}}\right)$,

for some $c \in \mathbb{R}$. Therefore, $\lambda \in L_{\text {loc }}^{2}([0, \infty) ; \mathbb{R})$ follows from $(5.12)$. Since $(u, v)^{T}$ solves (5.4) and $\lambda$ is given by (5.12), the computation from Step 1 can be reversed and yields that $(u, v, \lambda)$ satisfies $(5.10)$ and $\Psi\left((u, v)^{T}\right)=0$ holds.

Step 3: [Estimates] Let $(u, v, \lambda)$ be the unique solution. If (5.8) holds, Proposition 5.4 implies the exponential estimates (5.9) for $u$ and $v$. For $\lambda$, given by $(5.12)$, follows

$$
\begin{aligned}
\int_{0}^{\infty} e^{-c^{\prime} \tau}|\lambda(\tau)|^{2} d \tau \leq \mathrm{const} & \int_{0}^{\infty} e^{-c^{\prime} \tau}\left(\|u(\tau)\|_{H^{1}}^{2}+\|v(\tau)\|_{H^{1}}^{2}\right. \\
& \left.+\|G(\tau)\|_{L^{2}}^{2}+\left\|F_{1}(\tau)\right\|_{L^{2}}^{2}+\left\|F_{2}(\tau)\right\|_{L^{2}}^{2}\right) d \tau
\end{aligned}
$$

from the boundedness of $\Psi$ and $\Psi \circ P$. The integral converges for sufficiently large $c^{\prime}$ so that the estimate (5.9) for $\lambda$ follows. In the case of exponentially bounded inhomogeneities, the exponential boundedness of $\lambda$ again follows from (5.12) and the properties of $\Psi$ and $P$.

REMARK 5.7. (1) The proof shows that $\lambda$ is uniquely given by (5.12). This implies $\lambda \in \mathcal{C}([0, \infty))$ for $F_{1} \in \mathcal{C}\left([0, \infty) ;\left(H^{1}\right)^{\prime}\right), G \in \mathcal{C}\left([0, \infty) ; L^{2}\right)$, $F_{2} \in \mathcal{C}\left([0, \infty) ; L^{2}\right)$.

(2) Note that we did not make use of the spectral structure of $P$, i.e. Assumptions 1.3 and 4.1 , in the above proofs.

5.1.2. Proof of Linear Stability, Theorem 5.1. The stability proof now proceeds as in the purely hyperbolic case $[\mathbf{1 9}, \mathrm{Thm}$. 5.3]. Therefore we refer to that article and to $[\mathbf{1 8}]$ for the details and restrict here to the ideas and some differences originating from the parabolic-hyperbolic structure.

That the Laplace transform in combination with resolvent estimates can be use for the proof of stability is well known. We adapt several ideas from $[\mathbf{1 3}]$ to the PDAE problem considered here. Note that in that reference no PDAE problem was considered and also no justification for the Laplace transform is given. We justify its use in the following Step 1.

Step 1: Start with consistent initial data $u_{0}=0, v_{0}=0$, and $\lambda_{0}$. Assume that $F_{1}, G, F_{2}$ satisfy $(5.8)$ for $\eta_{L}=\delta$, and $\left(\left\|F_{1}(t)\right\|_{L^{2}}^{2}+\|G(t)\|_{L^{2}}^{2}+\left\|F_{2}(t)\right\|_{L^{2}}^{2}\right) \leq K_{e} e^{-\eta_{e} t}$ for some $K_{e}, \eta_{e} \in \mathbb{R}$. Proposition 5.6 shows exponential boundedness of the unique solution $(u, v, \lambda)^{T}$ of (4.2). Thus, its Laplace transform exists for all $s \in \mathbb{C}$ with 
Re $s>\alpha$ for some $\alpha \in \mathbb{R}$. As in the proof of [19, Thm. 5.3] the Laplace-transformed functions $(\hat{u}, \hat{v}, \hat{\lambda})^{T}$ holomorphically extend to the half plane $\{\operatorname{Re} s>-\delta\}$ and are given by the solution of the resolvent equation (4.3).

Propositions 4.13-4.15 show that for every $\eta_{0}<\delta$ there is a constant $K_{\eta_{0}}$, independent of $F_{1}, G, F_{2}$, so that for all $s \in \mathbb{C}$ with $\operatorname{Re} s \geq-\eta_{0}$ holds

$$
\|\hat{u}(s)\|_{H^{1}}^{2}+\|\hat{v}(s)\|_{H^{1}}^{2}+|\hat{\lambda}(s)|^{2} \leq K_{\eta_{0}}\left(\left\|\hat{F}_{1}(s)\right\|_{L^{2}}^{2}+\|\hat{G}(s)\|_{L^{2}}^{2}+\left\|\hat{F}_{2}(s)\right\|_{H^{1}}^{2}\right) .
$$

By assumption the right hand side is uniformly bounded for all $\operatorname{Re} s \geq-\eta_{0}$ and, therefore, by $[\mathbf{1}$, Thm. 4.4.13] the Laplace transforms of $u, v, \lambda$ exist for all $\operatorname{Re} s>$ $-\delta$ and coincide with $\hat{u}, \hat{v}, \hat{\lambda}$. This can be interpreted as a justification to shift the contour for the inverse Laplace transform into the left half plane. Therefore, Plancherel's Theorem [1, Thm. 1.8.2] shows for all $\eta \leq \eta_{0}$,

$$
\begin{aligned}
& \int_{0}^{\infty} e^{2 \eta \tau}\left(\|u(\tau)\|_{H^{1}}^{2}+\|v(\tau)\|_{H^{1}}^{2}+|\lambda(\tau)|^{2}\right) d \tau \\
& \leq K_{\eta_{0}} \int_{0}^{\infty} e^{2 \eta \tau}\left(\left\|F_{1}(\tau)\right\|_{L^{2}}^{2}+\|G(\tau)\|_{L^{2}}^{2}+\left\|F_{2}(\tau)\right\|_{H^{1}}^{2}\right) d \tau
\end{aligned}
$$

Step 2: Consider consistent initial data $\left(u_{0}, v_{0}\right)=0, \lambda_{0}$, and $F_{1} \in L_{l o c}^{\infty}\left([0, \infty) ; L^{2}\right)$, $G \in L_{l o c}^{\infty}\left([0, \infty) ; H^{1}\right), F_{2} \in L_{l o c}^{\infty}\left([0, \infty) ; H^{1}\right)$. A "future does not influence the past" argument similar to the one used in [19], proves with $\eta$ and $K_{\eta_{0}}$ from Step 1 for all $t \geq 0$

$$
\begin{aligned}
\int_{0}^{t} e^{2 \eta \tau}\left(\|u(\tau)\|_{H^{1}}^{2}+\right. & \left.\|v(\tau)\|_{H^{1}}^{2}+|\lambda(\tau)|^{2}\right) d \tau \\
& \leq K_{\eta_{0}} \int_{0}^{t} e^{2 \eta \tau}\left(\left\|F_{1}(\tau)\right\|_{L^{2}}^{2}+\|G(\tau)\|_{L^{2}}^{2}+\left\|F_{2}(\tau)\right\|_{H^{1}}^{2}\right) d \tau
\end{aligned}
$$

By only considering the PDE part of (4.2) with $\lambda W_{x}^{o}$ as part of the forcing, we obtain from the last estimate with the energy estimate, Lemma 2.6 , for all $t \geq 0$,

$$
\begin{aligned}
\|W(t)\|_{H^{1}}^{2} & +e^{-2 \eta t} \int_{0}^{t} e^{2 \eta \tau}\left(\|W(\tau)\|_{H^{1}}^{2}+|\lambda(\tau)|^{2}\right) d \tau \\
& \leq e^{-2 \eta t} C_{\eta_{0}} \int_{0}^{t} e^{2 \eta \tau}\left(\|G(\tau)\|_{H^{1}}^{2}+\left\|F_{1}(\tau)\right\|_{L^{2}}^{2}+\left\|F_{2}(\tau)\right\|_{H^{1}}^{2}\right) d t
\end{aligned}
$$

The constant $C_{\eta_{0}}$ does not depend on the choice of $\eta \leq \eta_{0}$.

Step 3: In case $W_{0}=\left(u_{0}, v_{0}\right)^{T} \neq 0 \in H^{2} \times H^{2}$, transform (4.2) via $\widetilde{W}:=$ $W-e^{-2 \delta t} W_{0}$ to homogeneous initial data. Then the inhomogeneities become

$$
\begin{aligned}
\widetilde{F}_{1}(\cdot, t) & =F_{1}(\cdot, t)+2 \delta e^{-2 \delta t} u_{0}+e^{-2 \delta t}\left(\left(D g^{o} W_{0}\right)_{x}+D f_{1}^{o} W_{0}\right), \\
\widetilde{G}(\cdot, t) & =G(\cdot, t)+e^{-2 \delta t} A_{11} u_{0, x} \\
\widetilde{F}_{2}(\cdot, t) & =F_{2}(\cdot, t)+2 \delta e^{-2 \delta t} v_{0}+e^{-2 \delta t}\left(B_{22} v_{0, x}+D f_{2}^{o} W_{0}\right)
\end{aligned}
$$


and have the same smoothness properties as $F_{1}, G, F_{2}$. The result from Step 2 now applies to the transformed variables and shows for all $t \geq 0$

$$
\begin{gathered}
\|W(t)\|_{H^{1}}^{2}+e^{-2 \eta t} \int_{0}^{t} e^{2 \eta \tau}\left(\|W(\tau)\|_{H^{1}}^{2}+|\lambda(\tau)|^{2}\right) d \tau \\
\leq 2\left\{\|\widetilde{W}(t)\|_{H^{1}}^{2}+e^{-2 \eta t} \int_{0}^{t} e^{2 \eta \tau}\left(\|\widetilde{W}(\tau)\|_{H^{1}}^{2}+|\lambda(\tau)|^{2}\right) d \tau\right. \\
\left.\quad+e^{-4 \delta t}\left\|W_{0}\right\|_{H^{1}}^{2}+e^{-2 \eta t} \int_{0}^{t} e^{(2 \eta-4 \delta) \tau}\left\|W_{0}\right\|_{H^{1}}^{2} d \tau\right\} \\
\leq 2\left\{e^{-2 \eta t} C_{\eta_{0}} \int_{0}^{t} e^{2 \eta \tau}\left(\left\|\widetilde{F}_{1}(\tau)\right\|_{L^{2}}^{2}+\|\widetilde{G}(t)\|_{H^{1}}^{2}+\left\|\widetilde{F}_{2}(\tau)\right\|_{H^{1}}^{2}\right) d \tau\right. \\
\left.\quad+e^{-2 \eta t}\left(1+\frac{1}{2 \delta}\right)\left[\left\|u_{0}\right\|_{H^{1}}^{2}+\left\|v_{0}\right\|_{H^{1}}^{2}\right]\right\}
\end{gathered}
$$

Furthermore, there is $\widetilde{C}$, independent of $W_{0}, \lambda_{0}, F_{1}, G, F_{2}$, so that for all $t \geq 0$ holds

$$
\begin{aligned}
\|\widetilde{G}(t)\|_{H^{1}} & \leq\|G(t)\|_{H^{1}}+\widetilde{C} e^{-2 \delta t}\left\|u_{0}\right\|_{H^{2}}, \\
\left\|\widetilde{F}_{1}(t)\right\|_{L^{2}} & \leq\left\|F_{1}(t)\right\|_{L^{2}}+\widetilde{C} e^{-2 \delta t}\left(\left\|u_{0}\right\|_{H^{1}}+\left\|v_{0}\right\|_{H^{1}}\right), \\
\left\|\widetilde{F}_{2}(t)\right\|_{H^{1}} & \leq\left\|F_{2}(t)\right\|_{H^{1}}+\widetilde{C} e^{-2 \delta t}\left(\left\|u_{0}\right\|_{H^{1}}+\left\|v_{0}\right\|_{H^{2}}\right) .
\end{aligned}
$$

Inserting these estimates finish the proof of Theorem 5.1.

REMARK 5.8. Note that in the last step of the proof we had to use $H^{2}$ estimates of the initial data to obtain an $H^{1}$ estimate for the solution.

5.2. Nonlinear Stability of the PDAE. In this section we prove stability for the nonlinear parabolic-hyperbolic PDAE (3.7a) subject to consistent initial data $\widetilde{W}(0)=\left(\widetilde{u}_{0}, \widetilde{v}_{0}\right)^{T}, \widetilde{\varphi}(0)=\widetilde{\varphi}_{0}, \widetilde{\lambda}(0)=\widetilde{\lambda}_{0}$.

TheOREM 5.9. Impose Assumptions 1.1, 1.2, 1.3, 3.1, 4.1. Then for every $0<\eta<\delta$, $\delta$ from Assumptions 1.3 and 4.1, there are $\rho_{0}, \theta_{0}>0$ so that for all consistent initial data $\widetilde{u}_{0}, \widetilde{v}_{0} \in H^{2}, \widetilde{\varphi}_{0}, \widetilde{\lambda}_{0} \in \mathbb{R}$, with $\left\|\widetilde{u}_{0}\right\|_{H^{2}}^{2}+\left\|\widetilde{v}_{0}\right\|_{H^{2}}^{2} \leq \rho_{0}^{2}$ and $\left|\widetilde{\varphi}_{0}\right| \leq \theta_{0}$, there is a unique solution $(\widetilde{u}, \widetilde{v}, \widetilde{\varphi}, \widetilde{\lambda})$ of $(3.7)$ on $[0, \infty)$. The solution satisfies $\widetilde{\varphi}(t) \in U$ for all $t \geq 0$, $U$ given by Lemma 3.2. Moreover, there is $\widetilde{\varphi}_{\infty} \in \mathbb{R}$ so that with $C_{l}$ from Theorem 5.1 hold for all $t \geq 0$,

$$
\begin{aligned}
\left|\widetilde{\varphi}_{\infty}\right| & \leq\left|\widetilde{\varphi}_{0}\right|+\left(\frac{C_{l}}{\eta}\right)^{1 / 2}\left(\left\|\widetilde{u}_{0}\right\|_{H^{2}}^{2}+\left\|\widetilde{v}_{0}\right\|_{H^{2}}^{2}\right)^{1 / 2}, \\
\left|\widetilde{\varphi}(t)-\widetilde{\varphi}_{\infty}\right|^{2} & \leq \frac{C_{l}}{\eta}\left(\left\|\widetilde{u}_{0}\right\|_{H^{2}}^{2}+\left\|\widetilde{v}_{0}\right\|_{H^{2}}^{2}\right) e^{-2 \eta t}, \\
\|\widetilde{u}(t)\|_{H^{1}}^{2}+\|\widetilde{v}(t)\|_{H^{1}}^{2} & \leq C_{l}\left(\left\|\widetilde{u}_{0}\right\|_{H^{2}}^{2}+\left\|\widetilde{v}_{0}\right\|_{H^{2}}^{2}\right) e^{-2 \eta t}, \\
\int_{0}^{t} e^{2 \eta \tau}|\widetilde{\lambda}(\tau)|^{2} d \tau & \leq 2 C_{l}\left(\left\|\widetilde{u}_{0}\right\|_{H^{2}}^{2}+\left\|\widetilde{v}_{0}\right\|_{H^{2}}^{2}\right) .
\end{aligned}
$$

The techniques are closely related to those used in $[\mathbf{2 0}]$ for the purely hyperbolic case. Therefore we refer to that article and to $[\mathbf{1 8}]$ at some places of the proof.

Proof. Step 0: [Rescaling] Let $\varepsilon>0$, $\varepsilon$ will be chosen below, and consider the rescaled variables $\varepsilon \bar{u}=\widetilde{u}, \varepsilon \bar{v}=\widetilde{v}, \varepsilon \bar{\varphi}=\widetilde{\varphi}, \varepsilon \bar{\lambda}=\widetilde{\lambda}$. Also define for $i, j=1,2$ 
the rescaled nonlinearities

$$
\begin{gathered}
\varepsilon^{2} F_{i j}^{\varepsilon}(\bar{\varphi}, \bar{u}, \bar{v}):=F_{i j}(\varepsilon \bar{\varphi}, \varepsilon \bar{u}, \varepsilon \bar{v}), \varepsilon^{2} G_{i}^{\varepsilon}(\bar{\varphi}, \bar{u}, \bar{v}):=G_{i}(\varepsilon \bar{\varphi}, \varepsilon \bar{u}, \varepsilon \bar{v}), \\
\varepsilon^{2} R_{i}^{\varepsilon}(\bar{\varphi}, \bar{\lambda}):=R_{i}(\varepsilon \bar{\varphi}, \varepsilon \bar{\lambda}) .
\end{gathered}
$$

where $F_{i j}, G_{i}, R_{i}$ are given in (3.8). This yields the rescaled PDAE

(5.19a)

$$
\begin{aligned}
\bar{u}_{t} & =A_{11} \bar{u}_{x x}+\left\{D g^{o}\left(\begin{array}{c}
\bar{u} \\
\bar{v}
\end{array}\right)\right\}_{x}+D f_{1}^{o}\left(\begin{array}{c}
\bar{u} \\
\bar{v}
\end{array}\right)+\bar{\lambda} u_{x}^{o} \\
& +\varepsilon\left\{G_{1}^{\varepsilon}(\bar{\varphi}, \bar{u}, \bar{v})+G_{2}^{\varepsilon}(\bar{\varphi}, \bar{u}, \bar{v})\right\}_{x}+\varepsilon F_{11}^{\varepsilon}(\bar{\varphi}, \bar{u}, \bar{v})+\varepsilon F_{12}^{\varepsilon}(\bar{\varphi}, \bar{u}, \bar{v})+\varepsilon R_{1}^{\varepsilon}(\bar{\varphi}, \bar{u}), \\
\bar{v}_{t} & =B_{22} \bar{v}_{x}+D f_{2}^{o}\left(\begin{array}{c}
\bar{u} \\
\bar{v}
\end{array}\right)+\bar{\lambda} v_{x}^{o}+\varepsilon F_{21}^{\varepsilon}(\bar{\varphi}, \bar{u}, \bar{v})+\varepsilon F_{22}^{\varepsilon}(\bar{\varphi}, \bar{u}, \bar{v})+\varepsilon R_{2}^{\varepsilon}(\bar{\varphi}, \bar{\lambda}), \\
\bar{\varphi}_{t} & =\bar{\lambda} \\
0 & =\Psi(\bar{u}, \bar{v}),
\end{aligned}
$$

subject to the, again consistent, initial conditions

$$
\bar{u}(0)=\bar{u}_{0}:=\frac{1}{\varepsilon} \widetilde{u}_{0}, \quad \bar{v}(0)=\bar{v}_{0}:=\frac{1}{\varepsilon} \widetilde{v}_{0}, \quad \bar{\varphi}(0)=\bar{\varphi}_{0}:=\frac{1}{\varepsilon} \widetilde{\varphi}_{0}, \quad \bar{\lambda}(0)=\bar{\lambda}_{0}:=\frac{1}{\varepsilon} \widetilde{\lambda}_{0} .
$$

Then $(\widetilde{u}, \widetilde{v}, \widetilde{\varphi}, \widetilde{\lambda})$ is a solution of $(3.7)$ if and only if $(\bar{u}, \bar{v}, \bar{\varphi}, \bar{\lambda})$ is a solution of (5.19). Hence, it suffices to show that for every $0<\eta<\delta$ there are $\varepsilon_{0}, \omega_{0}>0$ so that for all $0<\varepsilon \leq \varepsilon_{0}$ and all initial data $\bar{u}_{0} \in H^{2}, \bar{v}_{0} \in H^{2}, \bar{\varphi}_{0}, \bar{\lambda}_{0} \in \mathbb{R}$ with $\left\|\bar{u}_{0}\right\|_{H^{2}}^{2}+\left\|\bar{v}_{0}\right\|_{H^{2}}^{2} \leq \omega_{0}$ and $\left|\bar{\varphi}_{0}\right| \leq 1$, (5.19) has a unique classical solution $(\bar{u}, \bar{v}, \bar{\varphi}, \bar{\lambda})$ on $[0, \infty),|\bar{\varphi}(t)| \leq 2$ for all $t \geq 0$ and, moreover, there is $\bar{\varphi}_{\infty} \in \mathbb{R}$ so that for all $t \geq 0,(5.18)$ with $^{\sim}$ replaced by ${ }^{-}$holds.

From now on let $U, V, E, \Phi$ be as in Section 3. Let $0<\eta<\delta$ be given and let $C_{l}$ be the constant from Theorem 5.1. Let $\varepsilon_{1}>0$ with $\varepsilon_{1} \leq\left(2 C_{l}\right)^{-1 / 2}$, $B_{3 \varepsilon_{1}}(0) \subset U$.

Step 1: [A priori estimates] Let $0<\varepsilon<\varepsilon_{1}$ and assume there is a solution $(\bar{u}, \bar{v}, \bar{\varphi}, \bar{\lambda})$ of $(5.19)$ on $[0, T], T>0$, satisfying

$$
|\bar{\varphi}(t)| \leq 2, \quad\left(\|\bar{u}(t)\|_{H^{1}}^{2}+\|\bar{v}(t)\|_{H^{1}}^{2}\right)^{1 / 2} \leq 2 \quad \forall 0 \leq t \leq T .
$$

By Lemmas B.4, B.5, and B.6 the functions $F_{i j}^{\varepsilon}(\bar{\varphi}, \bar{u}, \bar{v}), G_{i}^{\varepsilon}(\bar{\varphi}, \bar{u}, \bar{v})$, and $R_{i}^{\varepsilon}(\bar{\varphi}, \bar{\lambda})$ are elements of $\mathcal{C}\left([0, T] ; H^{1}\right)$ and there is a constant $C_{n}$, independent of $T$, so that for all $0 \leq t \leq T$ :

$$
\begin{aligned}
\left\|G_{i}^{\varepsilon}(\bar{\varphi}, \bar{u}, \bar{v})\right\|_{H^{1}} & \leq C_{n}\left(\|\bar{u}\|_{H^{1}}^{2}+\|\bar{v}\|_{H^{1}}^{2}\right), & i & =1,2, \\
\left\|F_{i j}^{\varepsilon}(\bar{\varphi}, \bar{u}, \bar{v})\right\|_{H^{1}}^{2} & \leq C_{n}\left(\|\bar{u}\|_{H^{1}}^{2}+\|\bar{v}\|_{H^{1}}^{2}\right), & i, j & =1,2, \\
\left\|R_{i}^{\varepsilon}(\bar{\varphi}, \bar{\lambda})\right\|_{H^{1}}^{2} & \leq C_{n}|\bar{\lambda}|^{2}, & i & =1,2 .
\end{aligned}
$$

Consider the nonlinearities as inhomogeneities in the linear PDAE (4.1), i.e.

$$
G=\varepsilon\left(G_{1}^{\varepsilon}+G_{2}^{\varepsilon}\right), \quad F_{1}=\varepsilon\left(F_{11}^{\varepsilon}+F_{12}^{\varepsilon}+R_{1}^{\varepsilon}\right), \quad F_{2}=\varepsilon\left(F_{21}^{\varepsilon}+F_{22}^{\varepsilon}+R_{2}^{\varepsilon}\right) .
$$

Then Theorem 5.1 applies and, together with (5.20), yields for all $0 \leq t \leq T$,

$$
\begin{aligned}
& \|\bar{u}(t)\|_{H^{1}}^{2}+\|\bar{v}(t)\|_{H^{1}}^{2}+e^{-2 \eta t} \int_{0}^{t} e^{2 \eta \tau}\left(\|\bar{u}(\tau)\|_{H^{1}}^{2}+\|\bar{v}(\tau)\|_{H^{1}}^{2}+|\bar{\lambda}(\tau)|^{2}\right) d \tau \\
\leq & C_{l} e^{-2 \eta t}\left[\left\|\bar{u}_{0}\right\|_{H^{2}}^{2}+\left\|\bar{v}_{0}\right\|_{H^{2}}^{2}+5 \varepsilon^{2} C_{n} \int_{0}^{t} e^{2 \eta \tau}\left(\|\bar{u}(\tau)\|_{H^{1}}^{2}+\|\bar{v}(\tau)\|_{H^{1}}^{2}+|\bar{\lambda}(\tau)|^{2}\right) d \tau\right] .
\end{aligned}
$$


If $0<\varepsilon \leq \varepsilon_{0}=: \min \left(\varepsilon_{1},\left(10 C_{l} C_{n}\right)^{-1 / 2}\right)$, it follows for all $0 \leq t \leq T$ the bound

$$
\begin{gathered}
\|\bar{u}(t)\|_{H^{1}}^{2}+\|\bar{v}(t)\|_{H^{1}}^{2}+\frac{1}{2} e^{-2 \eta t} \int_{0}^{t} e^{2 \eta \tau}\left(\|\bar{u}(\tau)\|_{H^{1}}^{2}+\|\bar{v}(\tau)\|_{H^{1}}^{2}+|\bar{\lambda}(\tau)|^{2}\right) d \tau \\
\leq C_{l} e^{-2 \eta t}\left[\left\|\bar{u}_{0}\right\|_{H^{2}}^{2}+\left\|\bar{v}_{0}\right\|_{H^{2}}^{2}\right]
\end{gathered}
$$

Because of $\bar{\varphi}_{t}=\bar{\lambda}$, this also yields an estimate for the algebraic variable:

$$
|\bar{\varphi}(t)| \leq\left|\bar{\varphi}_{0}+\int_{0}^{t} \bar{\lambda}(\tau) d \tau\right| \leq\left|\bar{\varphi}_{0}\right|+\left(\frac{C_{l}}{\eta}\right)^{1 / 2}\left[\left\|\bar{u}_{0}\right\|_{H^{2}}^{2}+\left\|\bar{v}_{0}\right\|_{H^{2}}^{2}\right]^{1 / 2}, \text { for all } 0 \leq t \leq T \text {. }
$$

Step 2: [Local Existence and Uniqueness] Let $0<\varepsilon \leq \varepsilon_{0}$ with $\varepsilon_{0}$ from Step 1. Then $(\bar{u}, \bar{v}, \bar{\varphi}, \bar{\lambda})$ is a solution of (5.19) in $[0, T]$ if an only if $(\varepsilon \bar{u}, \varepsilon \bar{v}, \varepsilon \bar{\varphi}, \varepsilon \bar{\lambda})$ solves (3.7) in $[0, T]$ with the corresponding initial data. Therefore, if $\varepsilon \bar{\varphi}(t) \in U$ for all $t \in[0, T]$, Theorem 3.5 shows that $(\bar{u}, \bar{v}, \bar{\varphi}, \bar{\lambda})$ is a solution of (5.19) if and only if $(u, v)=\Xi(\varepsilon \bar{\varphi}, \varepsilon \bar{u}, \varepsilon \bar{v})$ solves the Cauchy problem (1.3) in $[0, T]$ with $u(0)=\varepsilon \bar{u}_{0}+u^{o}\left(\cdot-\varepsilon \bar{\varphi}_{0}\right)$ and $v(0)=\varepsilon \bar{v}_{0}+v^{o}\left(\cdot-\varepsilon \bar{\varphi}_{0}\right)$.

Let $\left(u^{*}, v^{*}\right)$ denote the unique global solution of (1.3) with these initial data. Let $\left[0, T^{*}\right), T^{*} \in(0, \infty]$, denote its interval of existence (Theorem 2.8). The consistency assumption, $\varepsilon \leq \varepsilon_{1}$, and $\left|\bar{\varphi}_{0}\right| \leq 1$, imply

$$
\Psi\left(u^{*}(0)-u^{o}, v^{*}(0)-v^{o}\right) \in V .
$$

Because $\left(u^{*}, v^{*}\right)$ is continuous into $H^{1}$, there is $0<T_{1}<T^{*}$, such that $\Psi\left(u^{*}(t)-\right.$ $\left.\underline{u}, v^{*}(t)-\underline{v}\right) \in V$ for all $0 \leq t \leq T_{1}$. This proves local existence and uniqueness.

Step 3: [Global Existence] Global existence follows by a simple bootstrapping argument. We refer to [20] where a similar argument was used for a purely hyperbolic problem.

Step 4: [Rate of convergence] The estimates (5.18) follow from the a priori bounds (5.21) and (5.22).

\section{Nonlinear Stability of Traveling Waves}

In this section we prove our main stability result. We begin with asymptotic stability with asymptotic phase for steady states. Stability of traveling waves is a simple corollary.

TheOREm 6.1 (Asymptotic stability with asymptotic phase). Consider (1.3) and impose Assumptions 1.1, 1.2, 1.3, 4.1. Then for every $0<\eta<\delta$ there is $\rho>0$ so that for all $u_{0} \in u^{o}+H^{2}\left(\mathbb{R}, \mathbb{R}^{n}\right)$ and $v_{0} \in v^{o}+H^{2}\left(\mathbb{R}, \mathbb{R}^{m}\right)$, with $\left(\left\|u_{0}-u^{o}\right\|_{H^{2}}^{2}+\right.$ $\left.\left\|v_{0}-v^{o}\right\|_{H^{2}}^{2}\right)^{1 / 2}<\rho$, exists a unique solution $(u, v)$ of $(1.3)$ on $[0, \infty)$, and for all $0<T<\infty$,

$$
\begin{aligned}
& u \in \mathcal{C}\left([0, T] ; u^{o}+H^{1}\right) \cap H^{1}\left(0, T ; u^{o}+L^{2}\right) \cap L^{2}\left(0, T ; u^{o}+H^{2}\right), \\
& v \in \mathcal{C}\left([0, T] ; v^{o}+H^{1}\right) \cap H^{1}\left(0, T ; v^{o}+L^{2}\right) .
\end{aligned}
$$

Moreover, there is $\varphi_{\infty}=\varphi_{\infty}\left(u_{0}, v_{0}\right) \in \mathbb{R}$ and $C=C(\eta)>0$, so that

$$
\begin{aligned}
& \left|\varphi_{\infty}\right| \leq C\left(\left\|u_{0}-u^{o}\right\|_{H^{2}}+\left\|v_{0}-v^{o}\right\|_{H^{2}}\right), \quad \text { and } \\
& \left\|u(t)-u^{o}\left(\cdot-\varphi_{\infty}\right)\right\|_{H^{1}}+\left\|v(t)-v^{o}\left(\cdot-\varphi_{\infty}\right)\right\|_{H^{1}} \\
& \leq C\left(\left\|u_{0}-u^{o}\right\|_{H^{2}}+\left\|v_{0}-v^{o}\right\|_{H^{2}}\right) e^{-\eta t} \quad \forall t \geq 0 .
\end{aligned}
$$


For the proof we rewrite the system in the form (1.8), using the nonlinear change of coordinates (1.7) which was analyzed in Section 3. This method of proof was first presented in [20] for the pure hyperbolic case. Because it is not difficult to adapt the proof from that paper to the current situation, we only show that Theorem 5.9 applies to system (1.8).

Proof. Let $0<\eta<\delta$ be given. Choose some $\Psi$ which satisfies Assumption 3.1 and let $E, U, V, \Phi$ be given as in Section 3. Let $\rho_{0}, \theta_{0}>0$ be the constants from Theorem 5.9. Then the mapping $(u, v) \mapsto \Phi \circ \Psi\left(u-u^{o}, v-v^{o}\right)$ is continuously differentiable in an open neighborhood of $\left(u^{o}, v^{o}\right)$ in $\left(u^{o}+H^{2}\right) \times\left(v^{o}+H^{2}\right)$. In particular, there are $\rho_{1}, C_{l i p}>0$ and $C_{l i p}>0$ so that for all $\left\|u-u^{o}\right\|_{H^{1}}^{2}+\| v-$ $v^{o} \|_{H^{1}}^{2} \leq \rho_{1}^{2}$ holds

$$
\left|\Phi\left(\Psi\left(u-u^{o}, v-v^{o}\right)\right)\right| \leq C_{l i p}\left(\left\|u-u^{o}\right\|_{H^{1}}^{2}+\left\|v-v^{o}\right\|_{H^{1}}^{2}\right)^{1 / 2} .
$$

Let

$$
\rho=\min \left(\rho_{1}, \frac{\theta_{0}}{C_{l i p}}, \frac{\rho_{0}^{2}}{2\left(1+C_{l i p}^{2}\left(\left\|u_{x}^{o}\right\|_{H^{2}}^{2}+\left\|v_{x}^{o}\right\|_{H^{2}}^{2}\right)\right)}\right) .
$$

By Theorem 3.5 the initial data of the PDAE reformulation are given by $(3.7 \mathrm{~b})$, i.e. $\widetilde{\varphi}_{0}=\Phi\left(\Psi\left(u_{0}-u^{o}, v_{0}-v^{o}\right)\right), \widetilde{u}_{0}=u_{0}-u^{o}\left(\cdot-\widetilde{\varphi}_{0}\right)$, and $\widetilde{v}_{0}=v_{0}-v^{o}\left(\cdot-\widetilde{\varphi}_{0}\right)$. Therefore, if $\left\|u_{0}-u^{o}\right\|_{H^{2}}^{2}+\left\|v_{0}-v^{o}\right\|_{H^{1}}^{2} \leq \rho^{2}$ it follows from (6.3)

$$
\left|\widetilde{\varphi}_{0}\right| \leq C_{l i p}\left(\left\|u_{0}-u^{o}\right\|_{H^{1}}^{2}+\left\|v_{0}-v^{o}\right\|_{H^{1}}^{2}\right)^{1 / 2} \leq \theta_{0} .
$$

Inserting (6.4) into the formulas for $\widetilde{u}_{0}$ and $\widetilde{v}_{0}$, shows

$$
\begin{gathered}
\leq 2\left(\left\|u_{0}-u^{o}\right\|_{H^{2}}^{2}+\left\|v_{0}-v^{o}\right\|_{H^{2}}^{2}+\left\|u^{o}-u^{o}\left(\cdot-\widetilde{\varphi}_{0}\right)\right\|_{H^{2}}^{2}+\left\|v^{o}-v^{o}\left(\cdot-\widetilde{\varphi}_{0}\right)\right\|_{H^{2}}^{2}\right) \\
\leq 2\left(1+\left(\left\|u_{x}^{o}\right\|_{H^{2}}^{2}+\left\|v_{x}^{o}\right\|_{H^{2}}^{2}\right) C_{l i p}^{2}\right)\left(\left\|u_{0}-u^{o}\right\|_{H^{2}}^{2}+\left\|v_{0}-v^{o}\right\|_{H^{2}}^{2}\right) \leq \rho_{0}^{2} .
\end{gathered}
$$

Therefore, Theorem 5.9 applies.

The assertion then easily follows from Theorem 3.5 and the convergence results (5.18a)-(5.18d). For the details we refere to the analysis of the hyperbolic case in $[\mathbf{2 0}]$.

By going into a co-moving frame, a traveling wave becomes a steady state. Therefore, Theorem 6.1 immediately imples the stability result for traveling waves, Theorem 1.4.

\section{Application to the Hodgkin-Huxley Model}

The nerve axon equations, as presented by Hodgkin and Huxley in [11], read

$$
\begin{aligned}
C_{M} V_{t} & =\frac{a}{2 R} V_{x x}-\bar{g}_{K} n^{4}\left(V-V_{K}\right)-\bar{g}_{N a} m^{3} h\left(V-V_{N a}\right)-\bar{g}_{l}\left(V-V_{l}\right), \\
n_{t} & =\alpha_{n}(V)(1-n)-\beta_{n}(V) n, \\
m_{t} & =\alpha_{m}(V)(1-m)-\beta_{m}(V) m, \\
h_{t} & =\alpha_{h}(V)(1-h)-\beta_{h}(V) h,
\end{aligned}
$$


where

$$
\begin{array}{lll}
\alpha_{n}=\frac{1}{100}(V+10)\left(\exp \left(\frac{V+10}{10}\right)-1\right)^{-1}, & \beta_{n}=\frac{1}{8} \exp \left(\frac{V}{80}\right), \\
\alpha_{m}=\frac{1}{10}(V+25)\left(\exp \left(\frac{V+25}{10}\right)-1\right)^{-1}, & \beta_{m}=4 \exp \left(\frac{V}{18}\right), \\
\alpha_{h}=\frac{7}{100} \exp \left(\frac{V}{20}\right), & \beta_{h}=\left(\exp \left(\frac{V+30}{10}\right)+1\right)^{-1} .
\end{array}
$$

The values of the constants in (7.1) are

$C_{M}=1, V_{N a}=-115, V_{K}=12, V_{l}=-10.613, \bar{g}_{N a}=120, \bar{g}_{K}=36, \bar{g}_{l}=0.3$, and $a, R$ are assumed to be larger than zero. Denote $u(x, t)=V(x, t) \in \mathbb{R}$ and $v(x, t)=(n(x, t), m(x, t), h(x, t))^{T} \in \mathbb{R}^{3}$ and define the functions

$$
\begin{aligned}
f_{1}(u, v)= & \frac{1}{C_{M}}\left(-\bar{g}_{K} n^{4}\left(V-V_{K}\right)-\bar{g}_{N a} m^{3} h\left(V-V_{N a}\right)-\bar{g}_{l}\left(V-V_{l}\right)\right) \\
f_{2}(u, v)= & \left(\begin{array}{c}
\alpha_{n}(u)\left(1-v_{1}\right)-\beta_{n}(u) v_{1} \\
\alpha_{m}(u)\left(1-v_{2}\right)-\beta_{m}(u) v_{2} \\
\alpha_{h}(u)\left(1-v_{3}\right)-\beta_{h}(u) v_{3}
\end{array}\right) .
\end{aligned}
$$

Then (7.1) can be written in the form (1.1):

$$
u_{t}=\frac{a}{2 R C_{M}} u_{x x}+f_{1}(u, v), \quad v_{t}=f_{2}(u, v)
$$

It is well-known, that the system has a traveling wave solution, whose profile and speed we denote by $\left(u^{o}, v^{o}\right)$ and $\lambda^{o}$, respectively. In the co-moving frame with speed $\lambda^{o}(7.2)$ reads

$$
u_{t}=\frac{a}{2 R C_{M}} u_{x x}+\lambda^{o} u_{x}+f_{1}(u, v), \quad v_{t}=\lambda^{o} v_{x}+f_{2}(u, v)
$$

By numerical calculation one finds a rest state for (7.3) at

$$
\left(u_{\infty}, v_{\infty}\right)=\left(V_{\infty}, n_{\infty}, m_{\infty}, h_{\infty}\right)=(-0.00362,0.31773,0.05295,0.59599),
$$

and $\left(u^{o}(x), v^{o}(x)\right) \rightarrow\left(u_{\infty}, v_{\infty}\right)$ as $x \rightarrow \pm \infty$. We check the Assumptions 1.1, 1.2, 1.3 and 4.1 which are needed for Theorem 1.4:

Assumption 1.1 obviously holds and also 4.11 is satisfied since $\lambda^{o} \neq 0$.

Consider part 3 of Assumption 4.1. Note that the coefficients of the linearized operator $P$ at $x= \pm \infty$ read (we drop the index \pm for readability reasons)

$$
\begin{gathered}
A=\left(\begin{array}{cc}
A_{11} & 0 \\
0 & 0
\end{array}\right)=\left(\begin{array}{c|c}
\frac{a}{2 R C_{M}} & 0 \cdots 0 \\
\hline 0 & \\
\vdots & 0 \\
0 &
\end{array}\right), \\
B=\left(\begin{array}{cc}
B_{11} & 0 \\
0 & B_{22}
\end{array}\right)=\left(\begin{array}{c|ccc}
\lambda^{o} & 0 \cdots 0 \\
\hline 0 & \vdots & \lambda^{o} I \\
0 &
\end{array}\right), \\
C=\left(c_{i j}\right)=\left(\begin{array}{l}
f_{1}^{\prime} \\
f_{2}^{\prime}
\end{array}\right)\left(\begin{array}{l}
u_{\infty} \\
v_{\infty}
\end{array}\right)=\left(\begin{array}{c|ccc}
-0.6775 & 55.44 & -69.19 & -2.049 \\
\hline-0.002808 & -0.1832 & 0 & 0 \\
-0.02637 & 0 & -4.223 & 0 \\
0.004107 & 0 & 0 & -0.1174
\end{array}\right)
\end{gathered}
$$




$$
=\left(\begin{array}{ll}
C_{11} & C_{12} \\
C_{21} & C_{22}
\end{array}\right)
$$

where we rounded $C$ to four digits. (Capital letters denote block matrices and small letters denote the matrix entries).

Let $H=\operatorname{diag}\left(1, h_{2}, h_{3}, h_{4}\right)=\operatorname{diag}\left(1,-\frac{c_{12}}{c_{21}}, 2000,-\frac{c_{14}}{c_{41}}\right)>0$. Then for every $v \in \mathbb{C}^{4}$ holds

$$
\begin{aligned}
& v^{*}\left(H C+C^{*} H\right) v \\
&=2 c_{11}\left|v_{1}\right|^{2}+2 h_{2} c_{22}\left|v_{2}\right|^{2}+2 h_{3} c_{33}\left|v_{3}\right|^{2}+2 h_{4} c_{44}\left|v_{4}\right|^{2}+2\left(c_{13}+h_{3} c_{31}\right) \operatorname{Re}\left(v_{1} v_{3}\right) \\
& \leq {\left[2 c_{11}-\frac{c_{13} h_{3} c_{31}}{100}\right]\left|v_{1}\right|^{2}+2 h_{2} c_{22}\left|v_{2}\right|^{2}+\left[2 h_{3} c_{33}-100\left(c_{13} h_{3} c_{31}\right)\right]\left|v_{3}\right|^{2}+2 h_{4} c_{44}\left|v_{4}\right|^{2} } \\
& \leq 2 \cdot(-0.05) v^{*} H v
\end{aligned}
$$

where we used $\left|\operatorname{Re}\left(v_{1} v_{3}\right)\right| \leq \frac{\varepsilon}{2}\left|v_{1}\right|^{2}+\frac{1}{2 \varepsilon}\left|v_{2}\right|^{2}$ with $\varepsilon=\frac{1}{100}$. Now assume $s \in$ $\sigma\left(-\omega^{2} A+i \omega B+C\right)$ for some $\omega \in \mathbb{R}$ and let $v \in \mathbb{C}^{4} \backslash\{0\}$ be a corresponding eigenvector, i.e. $\left(-\omega^{2} A+i \omega B+C\right) v=s v$. Therefore, $2 \operatorname{Re} s=2 \operatorname{Re}\left(\left(s v^{*} H v\right)\left(v^{*} H v\right)^{-1}\right)$, but

$$
\begin{aligned}
2 \operatorname{Re}\left(s v^{*} H v\right)=v^{*} H & \left(-\omega^{2} A+i \omega B+C\right) v+v^{*}\left(-\omega^{2} A^{*}-i \omega B+C^{*}\right) H v \\
& =-\omega^{2} \frac{a}{R C_{M}}\left|v_{1}\right|^{2}+v^{*}\left(H C+C^{*} H\right) v \leq 2 \cdot(-0.05) v^{*} H v
\end{aligned}
$$

implies Re $s \leq-0.05$, so that Assumption 4.13 holds with $\delta=0.05$.

To verify Assumption 1.2 and part 2 of Assumption 4.1, we consider the profile of the traveling wave. First of all, the coefficients $f_{1}, f_{2}$ and $g$ are sufficiently smooth. The profile $\left(u^{o}, v^{o}\right)$ is a steady state of (7.3) and we write the resulting infinite boundary value problem as a first order system by using the transformation $U=\left(U_{1}, U_{2}, U_{3: 5}\right)=\left(u, u_{x}, v\right)$. This yields

$$
U^{\prime}=\left(\begin{array}{c}
U_{2} \\
-\frac{\lambda^{o} 2 R C_{M}}{a} U_{2}-\frac{2 R C_{M}}{a} f_{1}\left(U_{1}, U_{3: 5}\right) \\
-\frac{1}{\lambda^{o}} f_{2}\left(U_{1}, U_{3: 5}\right)
\end{array}\right)
$$

and linearization at the fixed point $\left(u_{\infty}, 0, v_{\infty}\right)^{T}$ yields

$$
U^{\prime}=\left(\begin{array}{ccc}
0 & 1 & 0 \\
-A_{11}^{-1} C_{11} & -A_{11}^{-1} \lambda^{o} & -A_{11}^{-1} C_{12} \\
-\frac{1}{\lambda^{o}} C_{21} & 0 & -\frac{1}{\lambda^{o}} C_{22}
\end{array}\right) U=: M_{\infty} U .
$$

The above analysis implies that $M_{\infty}$ is hyperbolic: Assume $(u, w, v)^{T} \in \mathbb{C}^{1+1+3}$ is an eigenvector of $M_{\infty}$ to the eigenvalue $\kappa$, i.e. $M_{\infty}(u, w, v)^{T}=\kappa(u, w, v)^{T}$. This leads to

$$
\left(\kappa^{2}\left(\begin{array}{cc}
A_{11} & 0 \\
0 & 0
\end{array}\right)+\kappa\left(\begin{array}{cc}
B_{11} & 0 \\
0 & B_{22}
\end{array}\right)+\left(\begin{array}{ll}
C_{11} & C_{12} \\
C_{21} & C_{22}
\end{array}\right)\right)\left(\begin{array}{l}
u \\
v
\end{array}\right)=0 .
$$

Therefore, $0 \in \sigma\left(\kappa^{2} A+\kappa B+C\right)$, so that $\operatorname{Re} \kappa \neq 0$ since Assumption 4.13 . holds. Therefore, $M_{\infty}$ is hyperbolic and the solution $\left(u^{o}, u_{x}^{o}, v^{o}\right)$ approaches the rest state $\left(u_{\infty}, 0, v_{\infty}\right)$ exponentially fast. Hence $\left(u^{o}, v^{o}\right) \in \mathcal{C}_{b}^{1}$ and $\left(u_{x}^{o}, v_{x}^{o}\right) \in H^{2}$ follows, so that Assumption 1.2 and part 2 of 4.1 hold. Thus, all Assumptions of Theorem 1.4 except for Assumption 1.3 are verified.

THEOREM 7.1. A traveling pulse solution of the Hodgkin-Huxley model (7.1) with the asymptotic states $\left(u_{\infty}, v_{\infty}\right)$ is asymptotically stable if Assumption 1.3 holds. 
Note that Assumption 1.3 can checked numerically by approximation with a finite interval boundary value problem (see for example $[\mathbf{1 7}]$ ).

\section{Appendix A. A Perturbation Result from Linear Algebra}

We recall a well-known result about the perturbation of invariant subspaces. A reference is $[\mathbf{2 2}, \S \mathrm{V}$ Thm. 2.1]. Here we use $\|\cdot\|$ to denote the euclidean vector norm and its corresponding matrix norm. Consider a matrix written in block form:

$$
W=\left(\begin{array}{ll}
W_{11} & W_{12} \\
W_{21} & W_{22}
\end{array}\right) \in \mathbb{C}^{n+m, n+m}
$$

where $W_{11} \in \mathbb{C}^{n, n}, W_{12} \in \mathbb{C}^{n, m}, W_{21} \in \mathbb{C}^{m, n}, W_{22} \in \mathbb{C}^{m, m}$.

Theorem A.1. Let $W$ be given as above. Assume

$$
\delta=\operatorname{sep}\left(W_{11}, W_{22}\right):=\inf _{P \in \mathbb{C}^{m, n},\|P\|=1}\left\|P W_{11}-W_{22} P\right\|>0 .
$$

If $\frac{\left\|W_{12}\right\|\left\|W_{21}\right\|}{\delta^{2}}<\frac{1}{4}$ there is a unique solution $P \in \mathbb{C}^{m, n}$ of

$$
P W_{11}-W_{22} P+P W_{12} P=W_{21}
$$

satisfying

$$
\|P\| \leq \frac{2\left\|W_{21}\right\|}{\delta} .
$$

Using $P$, the solution of (A.2), $W$ is similar to the upper triangular block matrix

$$
\left(\begin{array}{cc}
I & 0 \\
-P & I
\end{array}\right) W\left(\begin{array}{cc}
I & 0 \\
P & I
\end{array}\right)=\left(\begin{array}{cc}
W_{11}+W_{12} P & W_{12} \\
0 & W_{22}-P W_{12}
\end{array}\right)
$$

Proof of Lemma 4.7. For convenience we suppress \pm in the proof. Let $W_{i j}$ be defined by

$$
\left(\begin{array}{ll}
W_{11}(\omega) & W_{12}(\omega) \\
W_{21}(\omega) & W_{22}(\omega)
\end{array}\right):=\left(\begin{array}{cc}
-\omega^{2} A_{11}+i \omega B_{11}+C_{11} & i \omega B_{12}+C_{12} \\
C_{21} & i \omega B_{22}+C_{22}
\end{array}\right)
$$

For the separation of the matrices $W_{11}(\omega)$ and $W_{22}(\omega)$ holds

$$
\begin{aligned}
& \operatorname{sep}\left(W_{11}(\omega), W_{22}(\omega)\right):=\inf _{\|P\|=1}\left\|P W_{11}(\omega)-W_{22}(\omega) P\right\| \\
& \quad \geq \inf _{\|P\|=1}\left\|\omega^{2} P A\right\|-|\omega|\left\|B_{11}\right\|-\left\|C_{11}\right\|-|\omega|\left\|B_{22}\right\|-\left\|C_{22}\right\| \\
& \quad \geq \frac{\omega^{2}}{\left\|A^{-1}\right\|}-|\omega|\left(\left\|B_{11}\right\|+\left\|B_{22}\right\|\right)-\left(\left\|C_{11}\right\|+\left\|C_{22}\right\|\right) \geq \frac{\omega^{2}}{2\left\|A^{-1}\right\|} \quad \forall|\omega|>\omega_{1} .
\end{aligned}
$$

Therefore,

$$
\frac{\operatorname{sep}\left(W_{11}(\omega), W_{22}(\omega)\right)^{2}}{4\left\|W_{21}(\omega)\right\|}>\frac{\omega^{4}}{8|\omega|\left\|A^{-1}\right\|^{2}\left(\left\|B_{12}\right\|+\left\|C_{12}\right\|\right)} \forall \omega \in \mathbb{R},|\omega|>\omega_{1},
$$

so that there is $\omega_{2} \geq \omega_{1}$ such that for all $\omega \in \mathbb{R}$ with $|\omega|>\omega_{2}$

$$
\left\|C_{21}\right\|=\left\|W_{21}(\omega)\right\|<\frac{\operatorname{sep}\left(W_{11}(\omega), W_{22}(\omega)\right)^{2}}{4\left\|W_{21}(\omega)\right\|} .
$$

Then Theorem A.1 shows that for all such $\omega$ there is $P=P(\omega)$ so that

$$
\left(\begin{array}{ll}
W_{11} & W_{12} \\
W_{21} & W_{22}
\end{array}\right)\left(\begin{array}{ll}
I & 0 \\
P & I
\end{array}\right)=\left(\begin{array}{cc}
I & 0 \\
P & I
\end{array}\right)\left(\begin{array}{cc}
W_{11}+W_{12} P & W_{12} \\
0 & W_{22}-P W_{12}
\end{array}\right)
$$


what implies for the spectrum

$$
\sigma\left(\begin{array}{ll}
W_{11}(\omega) & W_{12}(\omega) \\
W_{21}(\omega) & W_{22}(\omega)
\end{array}\right)=\sigma\left(W_{11}(\omega)+W_{12}(\omega) P(\omega)\right) \cup \sigma\left(W_{22}(\omega)-P(\omega) W_{12}(\omega)\right)
$$

Furthermore, the matrix $P$ satisfies

$$
\|P(\omega)\|<2 \frac{\left\|C_{21}\right\|}{\operatorname{sep}\left(W_{11}(\omega), W_{22}(\omega)\right)} \leq \frac{4\left\|A^{-1}\right\|\left\|C_{21}\right\|}{\omega^{2}}
$$

and $P(\omega) W_{12}(\omega)$ is a perturbation of order $\frac{1}{|\omega|}$ of $i \omega B_{22}+C_{22}$. This implies the assertion (see for example [19, Lem. A.1].

We also use the perturbation result Theorem A.1 to obtain statements about the asymptotics of the matrices $M_{ \pm}(s)$ from (4.6) for $s \rightarrow+\infty$. For simplicity we suppress \pm .

Let $s \in \mathbb{R}$ and $s>>$. For $\rho=\sqrt{s}$ let

$$
S_{\rho}=\left(\begin{array}{ccc}
I & 0 & 0 \\
0 & \rho I & 0 \\
0 & 0 & I
\end{array}\right) \quad \text { and } \quad T_{B_{12}}=\left(\begin{array}{ccc}
I & 0 & 0 \\
0 & I & B_{12} \\
0 & 0 & I
\end{array}\right) \in \mathbb{C}^{n+n+m, n+n+m},
$$

obviously $S_{\rho}^{-1}=S_{\rho^{-1}}, T_{B_{12}}^{-1}=T_{-B_{12}}$. The matrix $M(s)$ is equivalent to

$$
\begin{aligned}
\widetilde{M}(s)=\left(\begin{array}{cc|c}
\widetilde{M}_{11}(s) & \widetilde{M}_{12}(s) \\
\widetilde{M}_{21}(s) & \widetilde{M}_{22}(s)
\end{array}\right):=S_{\rho^{-1}} T_{B_{12}} M(s) T_{-B_{12}} S_{\rho} \\
\quad=\left(\begin{array}{ccc}
0 & \rho A_{11}^{-1} & -A_{11}^{-1} B_{12} \\
\frac{1}{\rho}\left(s I-C_{11}\right) & -B_{11} A_{11}^{-1} & \frac{1}{\rho}\left(B_{11} A_{11}^{-1} B_{12}-C_{12}\right) \\
\hline-B_{22}^{-1} C_{21} & 0 & B_{22}^{-1}\left(s I-C_{22}\right)
\end{array}\right) .
\end{aligned}
$$

It is easy to see for sufficiently large $s \in \mathbb{R}$ that the separation of the diagonal blocks satisfies

$$
\delta=\operatorname{sep}\left(\widetilde{M}_{11}(s), \widetilde{M}_{22}(s)\right) \geq \inf _{\|P\|=1}\left\|s B_{22}^{-1} P\right\|-\rho c\|P\| \geq c \rho^{2}\|P\|
$$

where $c$ is a generic constant. Moreover, $\left\|\widetilde{M}_{12}(s)\right\|,\left\|\widetilde{M}_{21}(s)\right\| \leq c$, so that

$$
\frac{\left\|\widetilde{M}_{12}(s)\right\|\left\|\widetilde{M}_{21}(s)\right\|}{\delta^{2}} \leq c \rho^{-4}<\frac{1}{4}
$$

for $s$ sufficiently large. By Theorem A.1 there is a unique solution $P(s)$ of

$$
P(s) \widetilde{M}_{11}(s)-\widetilde{M}_{22}(s) P(s)+P(s) \widetilde{M}_{12}(s) P(s)=\widetilde{M}_{21}(s), \text { with }
$$

$$
\|P\| \leq 2 \rho^{-2}\left\|M_{21}(s)\right\| \leq c \rho^{-2} .
$$

This transforms $\widetilde{M}(s)$ into upper block triangular form $\widetilde{M}^{\prime}(s)$ via (A.4). Hence, it suffices to consider the spectra of

$$
\begin{aligned}
& \widetilde{M}_{11}(s)+\widetilde{M}_{12}(s) P(s)=\widetilde{M}_{11}(s)+\mathcal{O}\left(\frac{1}{\rho}\right)=\left(\begin{array}{cc}
0 & \rho A_{11}^{-1} \\
\rho I & 0
\end{array}\right)+\mathcal{O}(1), \\
& \widetilde{M}_{22}(s)-P(s) \widetilde{M}_{12}(s)=B_{22}^{-1}\left(s I-C_{22}\right)+\mathcal{O}\left(\frac{1}{\rho}\right)=s B_{22}^{-1}+\mathcal{O}(1),
\end{aligned}
$$


which are the diagonal blocks of $\widetilde{M^{\prime}}(s)$. Let $A_{11} T=T J$, where $J$ is a Jordan matrix. By Assumption 1.1 all eigenvalues of $J$ have positive real part and the positive square root $J^{\frac{1}{2}}$ is well defined. Then

$$
\left(\begin{array}{cc}
0 & A \\
I & 0
\end{array}\right)\left(\begin{array}{cc}
T J^{\frac{1}{2}} & -T J^{\frac{1}{2}} \\
T & T
\end{array}\right)=\left(\begin{array}{cc}
T J^{\frac{1}{2}} & -T J^{\frac{1}{2}} \\
T & T
\end{array}\right)\left(\begin{array}{cc}
J^{\frac{1}{2}} & 0 \\
0 & -J^{\frac{1}{2}}
\end{array}\right)=: \widetilde{T}\left(\begin{array}{cc}
J^{\frac{1}{2}} & 0 \\
0 & -J^{\frac{1}{2}}
\end{array}\right)
$$

so that

$$
\widetilde{T}^{-1}\left(\left(\begin{array}{cc}
0 & \rho A^{-1} \\
\rho I & 0
\end{array}\right)+\mathcal{O}(1)\right) \widetilde{T}=\rho\left(\begin{array}{cc}
J^{\frac{1}{2}} & 0 \\
0 & -J^{\frac{1}{2}}
\end{array}\right)+\mathcal{O}(1) .
$$

For $s$ sufficiently large, Gershgorin's Theorem (e.g. [24, p. 71]) implies that $\widetilde{M}_{11}(s)+$ $\widetilde{M}_{12}(s) P(s)$ has $n$ eigenvalues with real part larger than 0 and $n$ eigenvalues with real part less than 0 , counted with multiplicity. The invertibility of $B_{22}$ shows that the real diagonal matrix $B_{22}^{-1}$ is hyperbolic. Let $r$ denote the number of negative eigenvalues. Hence Gershgorin's Theorem implies for $s$ sufficiently large for $s B_{22}^{-1}+$ $\mathcal{O}(1)$ that there are $r$ eigenvalues with negative real part and $m-r$ eigenvalues with positive real part. Since $\widetilde{M}_{ \pm}^{\prime}(s)$ and $M_{ \pm}(s)$ are similar, this discussion proves:

Proposition A.2. There is $s_{0}$ so that for all $s \in \mathbb{R}, s \geq s_{0}$ the dimension of the stable subspaces of $M_{+}(s)$ and $M_{-}(s)$ coincide.

\section{Appendix B. Properties of Nonlinear Terms}

For reference purpose, we first collect some well-known facts about Nemytskii operators. Proofs can be found in [18, App. D].

Lemma B.1. Let $f \in \mathcal{C}^{3}\left(\mathbb{R}^{l}, \mathbb{R}^{m}\right), u^{o} \in \mathcal{C}_{b}^{2}\left(\mathbb{R}, \mathbb{R}^{l}\right)$ with $u_{x}^{o} \in H^{1}$. Then $u \in H^{1}$ implies

(1) $f\left(u^{o}+u\right)-f\left(u^{o}\right) \in H^{1}$,

(2) the well-known Taylor formula holds as an equality in $H^{1}$ :

$$
f\left(u^{o}+u\right)-f\left(u^{o}\right)=f_{u}\left(u^{o}\right)+\int_{0}^{1}(1-s) f_{u u}\left(u^{o}+s u\right) d s u^{2},
$$

(3) $f\left(u^{o}\right) \in L^{2}$ implies $f\left(u^{o}+u\right) \in H^{1}$.

Corollary B.2. If $u \in L^{2}\left(0, T ; H^{1}\right) \cap L^{\infty}\left(0, T ; H^{1}\right)$, then Taylor's formula (B.1) holds in $L^{2}\left(0, T ; H^{1}\right)$ and in particular it holds for a.e. $t \in[0, T]$ as an equality in $H^{1}$.

Corollary B.3. If $u \in \mathcal{C}\left([0, T] ; H^{1}\left(\mathbb{R}, \mathbb{R}^{l}\right)\right)$, then

$$
f\left(u^{o}+u\right)-f\left(u^{o}\right) \in \mathcal{C}\left([0, T] ; H^{1}\left(\mathbb{R}, \mathbb{R}^{m}\right)\right) .
$$

We now give some properties for the nonlinear terms (3.8), appearing in (5.19a). These are used to establish (5.20). Without mentioning it again, we assume in the following lemmas

$$
(u, v) \in \mathcal{C}\left([0, T] ; H^{1}\left(\mathbb{R}, \mathbb{R}^{n+m}\right)\right), \quad \varphi \in \mathcal{C}([0, T] ; \mathbb{R}), \quad \lambda \in \mathcal{C}([0, T] ; \mathbb{R}) .
$$

The results can be proved by showing the asserted estimates for $\mathcal{C}_{0}^{\infty}$-functions and then using the fact that $u$ and $v$ can be approximated by such functions. For details we refer to $[\mathbf{1 8}]$. 
Lemma B.4. For $\varepsilon>0$ the functions $\mathbb{R} \ni t \mapsto H(\varphi(t), u(t), v(t)), H \in$ $\left\{G_{1}^{\varepsilon}, F_{11}^{\varepsilon}, F_{21}^{\varepsilon}\right\}$, are elements of $\mathcal{C}\left([0, T] ; H^{1}\right)$. Moreover, there is $C>0$, depending on $g, f_{1}, f_{2}, u^{o}, v^{o}$, but independent of $\varepsilon$, so that

$\|H(\varphi(t), u(t), v(t))\|_{*}^{2} \leq C|\varphi(t)|^{2}\left(\|u(t)\|_{*}^{2}+\|v(t)\|_{*}^{2}\right), * \in\left\{L^{2}\left(\mathbb{R}, \mathbb{R}^{m}\right), H^{1}\left(\mathbb{R}, \mathbb{R}^{m}\right)\right\}$,

The following lemma looks very similar, but note that here the $(u, v)$ terms appear quadratic in the function.

Lemma B.5. Assume $0<\varepsilon \leq \varepsilon_{1}$ and $u, v$ as above with $\|(u, v)\|_{L^{\infty}\left([0, T] ; H^{1}\right)} \leq$ $K$. Then the functions $\mathbb{R} \ni t \mapsto H(\varphi(t), v(t)) \in H^{1}, H \in\left\{G_{2}^{\varepsilon}, F_{12}^{\varepsilon}, F_{22}^{\varepsilon}\right\}$, are elements of $\mathcal{C}\left([0, T] ; H^{1}\right)$. Furthermore, there is $C>0$, depending on $g, f_{1}, f_{2}, u^{o}, v^{o}, \varepsilon_{1}$, but independent of $\varepsilon$, so that

$\|H(\varphi(t), v(t))\|_{*}^{2} \leq C\left(\|u(t)\|_{H^{1}}^{2}+\|v(t)\|_{H^{1}}^{2}\right)\left(\|u(t)\|_{*}^{2}+\|v(t)\|_{*}^{2}\right), \quad * \in\left\{L^{2}, H^{1}\right\}$.

Lemma B.6. For every $\varepsilon>0$ the function $t \mapsto R^{\varepsilon}(\varphi(t), \lambda(t))$ is an element of $\mathcal{C}\left([0, T] ; H^{1}\right)$ and there is $C>0$, independent of $\varphi, \lambda, \varepsilon$ so that

$$
\left\|R^{\varepsilon}(\varphi(t), \lambda(t))\right\|_{H^{1}}^{2} \leq C|\varphi(t)|^{2}|\lambda(t)|^{2} .
$$

\section{Appendix C. Exponential Dichotomies}

In this appendix we recall the definition and some properties of exponential dichotomies (ED). Basic references are [7] and [16]. Let $L$ denote an ordinary differential operator

$$
L z=z_{x}-M(x) z, \quad x \in J,
$$

$M \in \mathcal{C}\left(J, \mathbb{C}^{l, l}\right)$ is continuous on the closed (but possibly unbounded) interval $J$. Let $\mathcal{S}(\cdot, \cdot)$ denote the solution-operator for $L$.

DeFinition C.1. The operator $L$ has an exponential dichotomy on $J$ if there are $K, \beta>0$, and for every $x \in J$ there is a projector $\pi(x) \in \mathbb{C}^{l, l}$ such that

$$
\begin{aligned}
S(x, y) \pi(y) & =\pi(x) S(x, y) & & \forall x, y \in J, \\
|S(x, y) \pi(y)| & \leq K e^{-\beta(x-y)} & & \forall x \geq y \in J, \\
|S(x, y)(I-\pi(y))| & \leq K e^{-\beta(y-x)} & & \forall x<y \in J .
\end{aligned}
$$

The triple $(K, \beta, \pi)$ is called the data of the dichotomy.

Linear boundary value problems where the differential operator has an ED satisfy solution estimates in terms of the dichotomy data.

Theorem C.2 ([4, Theorem A.1]). Let L have an ED on $J$ with data $(K, \beta, \pi)$. Define the Green's function $G$ with respect to $\pi$ for all $x, y \in J$ by

$$
G(x, y)= \begin{cases}S(x, y) \pi(y), & y \leq x, \\ S(x, y)(\pi(y)-I), & x<y .\end{cases}
$$

Then for every $r \in L^{2}\left(J, \mathbb{C}^{l}\right), \gamma_{-} \in \mathcal{R}\left(\pi\left(x_{-}\right)\right), \gamma_{+} \in \mathcal{R}\left(I-\pi\left(x_{+}\right)\right)$, there is a unique solution $z \in H^{1}\left(J, \mathbb{C}^{l}\right)$ of the boundary value problem

$$
\begin{gathered}
L z=r, \quad \text { in } L^{2}\left(J, \mathbb{C}^{l}\right), \\
\pi\left(x_{-}\right) z\left(x_{-}\right)=\gamma_{-}, \quad\left(I-\pi\left(x_{+}\right)\right) z\left(x_{+}\right)=\gamma_{+} .
\end{gathered}
$$


The solution is given by $z=z_{\text {sp }}+z_{\text {hom }}$, where

$$
\begin{aligned}
z_{s p}(x) & =\int_{J} G(x, y) r(y) d y \quad \text { is a solution of }(\text { C.3 }) \text { with } \gamma_{ \pm}=0, \text { and } \\
z_{\text {hom }}(x) & =S\left(x, x_{-}\right) \gamma_{-}+S\left(x, x_{+}\right) \gamma_{+} .
\end{aligned}
$$

Moreover, the function $z$ satisfies the estimates

$$
\begin{aligned}
\text { (C.6) } \quad \beta^{2}\left\|z_{s p}\right\|^{2}+\beta\left(\left|z_{s p}\left(x_{-}\right)\right|^{2}+\left|z_{s p}\left(x_{+}\right)\right|^{2}\right) & \leq 5 K^{2}\|r\|^{2}, \\
\text { (C.7) } \quad \beta\left\|z_{h o m}\right\|^{2}+\left(\left|z_{h o m}\left(x_{-}\right)\right|^{2}+\left|z_{h o m}\left(x_{+}\right)\right|^{2}\right) & \leq\left(2+3 K^{2}\right)\left(\left|\gamma_{-}\right|^{2}+\left|\gamma_{+}\right|^{2}\right) .
\end{aligned}
$$

In the case of unbounded $J$, the boundary conditions at $\pm \infty$ are part of the function space and not stated explicitly. In particular, the corresponding $\gamma_{ \pm}$are zero in (C.5) and (C.7).

An important property of EDs is its roughness under perturbations, which is stated in the next Theorem (cf. [4, Thm. A.3]).

Theorem C.3 (Roughness). Let $L$ have an (ED) on $J$ with data $(K, \beta, \pi)$. Assume $\Delta \in \mathcal{C}\left(J, \mathbb{C}^{l, l}\right)$ can be estimated by $3 K\|\Delta\|_{\infty}<\beta$.

Then the operator $\widetilde{L} z=z_{x}-(M+\Delta) z$ has an (ED) on $J$, too. The data $(\widetilde{K}, \widetilde{\beta}, \widetilde{\pi})$ can be chosen so that

$$
\begin{gathered}
\widetilde{K}=K\left(2+\frac{4\|\Delta\|_{\infty} K}{\beta-3\|\Delta\|_{\infty} K}\right), \quad \widetilde{\beta}=\beta-2\|\Delta\|_{\infty} K, \\
|\widetilde{\pi}(x)-\pi(x)| \leq K \widetilde{K} \int_{J} e^{-(\beta+\widetilde{\beta})|x-y|}|\Delta(y)| d y .
\end{gathered}
$$

The next lemma relates Fredholm properties to properties of the ED.

Lemma C.4 $([\mathbf{1 6}, \mathbf{5}])$. Let $M \in \mathcal{C}_{b}\left(\mathbb{R}, \mathcal{C}^{l, l}\right)$ such that for $L$ from (C.1) has EDs on $(-\infty, 0]$ and $[0, \infty)$ with data $\left(K_{ \pm}, \beta_{ \pm}, \pi_{ \pm}\right)$. Then $L: H^{1}\left(\mathbb{R}, \mathbb{C}^{l}\right) \rightarrow L^{2}\left(\mathbb{R}, \mathbb{C}^{l}\right)$ is Fredholm of index $\operatorname{dim}\left(\operatorname{span}\left(\pi_{+}(0)\right)\right)+\operatorname{dim}\left(\mathcal{N}\left(\pi_{-}(0)\right)\right)-l$.

\section{References}

[1] W. Arendt, C. J. K. Batty, M. Hieber, and F. Neubrander. Vector-valued Laplace transforms and Cauchy problems, volume 96 of Monographs in Mathematics. Birkhäuser Verlag, Basel, 2001.

[2] P. W. Bates and C. K. R. T. Jones. Invariant manifolds for semilinear partial differential equations. In Dynamics reported, Vol. 2, volume 2 of Dynam. Report. Ser. Dynam. Systems Appl., pages 1-38. Wiley, Chichester, 1989.

[3] W.-J. Beyn. The numerical computation of connecting orbits in dynamical systems. IMA J. Numer. Anal., 10(3):379-405, 1990.

[4] W.-J. Beyn and J. Lorenz. Stability of traveling waves: dichotomies and eigenvalue conditions on finite intervals. Numer. Funct. Anal. Optim., 20(3-4):201-244, 1999.

[5] W.-J. Beyn and J. Lorenz. Stability of viscous profiles: proofs via dichotomies. J. Dynam. Differential Equations, 18(1):141-195, 2006.

[6] W.-J. Beyn and J. Rottmann-Matthes. Resolvent estimates for boundary value problems on large intervals via the theory of discrete approximations. Numer. Funct. Anal. Optim., 28(5-6):603-629, 2007.

[7] W. A. Coppel. Dichotomies in stability theory, volume 629 of Lecture Notes in Mathematics. Springer-Verlag, Berlin, 1978.

[8] L. Evans. Partial differential equations, volume 19 of Graduate Studies in Mathematics. AMS, Providence, RI, 1998.

[9] A. Ghazaryan, Y. Latushkin, S. Schecter, and A. J. de Souza. Stability of gasless combustion fronts in one-dimensional solids. Arch. Ration. Mech. Anal., 198(3):981-1030, 2010. 
[10] D. Henry. Geometric theory of semilinear parabolic equations, volume 840 of Lecture Notes in Mathematics. Springer-Verlag, Berlin, 1981.

[11] A. L. Hodgkin and A. F. Huxley. A quantitative description of membrane current and its application to conduction and excitation in nerve. J. Physiol., 117:500-544, 1952.

[12] L. Hörmander. Lectures on nonlinear hyperbolic differential equations, volume 26 of Mathématiques \&s Applications (Berlin). Springer-Verlag, Berlin, 1997.

[13] G. Kreiss, H.-O. Kreiss, and N. A. Petersson. On the convergence of solutions of nonlinear hyperbolic-parabolic systems. SIAM J. Numer. Anal., 31(6):1577-1604, 1994.

[14] H.-O. Kreiss and J. Lorenz. Initial-boundary value problems and the Navier-Stokes equations, volume 136 of Pure and Applied Mathematics. Academic Press Inc., Boston, MA, 1989.

[15] H.-O. Kreiss and J. Lorenz. Stability for time-dependent differential equations. In Acta numerica, 1998, volume 7 of Acta Numer., pages 203-285. Cambridge Univ. Press, Cambridge, 1998.

[16] K. J. Palmer. Exponential dichotomies and transversal homoclinic points. J. Differential Equations, 55(2):225-256, 1984.

[17] J. Rottmann-Matthes. Spectral analysis of coupled hyperbolic-parabolic systems on finite and infinite intervals. In S. Benzoni-Gavage and D. Serre, editors, Hyperbolic problems: Theory, numerics and applications., pages 901-909, Berlin, 2007. Springer.

[18] J. Rottmann-Matthes. Computation and Stability of Patterns in Hyperbolic-Parabolic Systems. Shaker Verlag, Aachen, 2010. Ph.D. thesis, Bielefeld University.

[19] J. Rottmann-Matthes. Linear stability of traveling waves in nonstrictly hyperbolic pdes. $J$. Dynam. Differential Equations, 23(2):365-393, 2011.

[20] J. Rottmann-Matthes. Stability and freezing of nonlinear waves in first-order hyperbolic PDEs. Preprint 11-016, CRC 701, Bielefeld University, 2011.

[21] J. Rottmann-Matthes. Stability and freezing of waves in nonlinear hyperbolic-parabolic systems. Preprint 11-079, CRC 701, Bielefeld University, 2011.

[22] G. W. Stewart and J. G. Sun. Matrix perturbation theory. Computer Science and Scientific Computing. Academic Press Inc., Boston, MA, 1990.

[23] A. I. Volpert and S. I. Hudjaev. On the chauchy problem for composite systems of nonlinear differential equations. Math. USSR-Sb., 16(4):517-544, 1972.

[24] J. H. Wilkinson. The algebraic eigenvalue problem. Clarendon Press, Oxford, 1965.

[25] S. Wu, H. Chen, and W. Li. The local and global existence of the solutions of hyperbolicparabolic system modeling biological phenomena. Acta Math. Sci. Ser. B Engl. Ed., 28(1):101-116, 2008.

[26] K. Yosida. Functional analysis. Springer-Verlag, Berlin, fifth edition, 1978. Grundlehren der Mathematischen Wissenschaften, Band 123.

[27] S. Zheng. Nonlinear parabolic equations and hyperbolic-parabolic coupled systems, volume 76 of Pitman Monographs and Surveys in Pure and Applied Mathematics. Longman Scientific \& Technical, Harlow, 1995.

Department of Mathematics, Bielefeld University, P.O. Box 100131, 33501 BieleFELD 